\title{
THE MAGELLANIC STREAM: BREAK-UP AND ACCRETION ONTO THE HOT GALACTIC CORONA
}

\author{
Thor Tepper-García ${ }^{1}$, Joss Bland-Hawthorn ${ }^{1}$, and Ralph S. Sutherland ${ }^{2}$ \\ ${ }^{1}$ Sydney Institute for Astronomy, School of Physics, University of Sydney, NSW 2006, Australia \\ ${ }^{2}$ Mount Stromlo Observatory, Australia National University, Woden, ACT 2611, Australia \\ Received 2015 May 6; accepted 2015 September 22; published 2015 November 3
}

\begin{abstract}
The Magellanic $\mathrm{H}_{\mathrm{I}}$ Stream $\left(\approx 2 \times 10^{9} M_{\odot}[d / 55 \mathrm{kpc}]^{2}\right)$ encircling the Galaxy at a distance $d$ is arguably the most important tracer of what happens to gas accreting onto a disk galaxy. Recent observations reveal that the Stream's mass is in fact dominated $(3: 1)$ by its ionized component. Here we revisit the origin of the mysterious $\mathrm{H} \alpha$ recombination emission observed along much of its length that is overly bright $(\sim 150-200 \mathrm{mR})$ for the known Galactic ultraviolet (UV) background $\left(\approx 20-40 \mathrm{mR}[d / 55 \mathrm{kpc}]^{-2}\right)$. In an earlier model, we proposed that a slow shock cascade was operating along the Stream due to its interaction with the extended Galactic hot corona. We find that for a smooth coronal density profile, this model can explain the bright $\mathrm{H} \alpha$ emission if the coronal density satisfies $2 \times 10^{-4}<\left(n / \mathrm{cm}^{-3}\right)<4 \times 10^{-4}$ at $d=55 \mathrm{kpc}$. But in view of updated parameters for the Galactic halo and mounting evidence that most of the Stream must lie far beyond the Magellanic Clouds $(d>55 \mathrm{kpc})$, we revisit the shock cascade model in detail. At lower densities, the H i gas is broken down by the shock cascade but mostly mixes with the hot corona without significant recombination. At higher densities, the hot coronal mass (including the other baryonic components) exceeds the baryon budget of the Galaxy. If the H $\alpha$ emission arises from the shock cascade, the upper limit on the smooth coronal density constrains the Stream's mean distance to $\lesssim 75 \mathrm{kpc}$. If, as some models indicate, the Stream is even further out, either the shock cascade is operating in a regime where the corona is substantially mass-loaded with recent gas debris, or an entirely different ionization mechanism is responsible.
\end{abstract}

Key words: galaxies: evolution - galaxies: interactions - hydrodynamics - instabilities - Magellanic Clouds shock waves

\section{INTRODUCTION}

The Galaxy is surrounded by a vast amount of neutral gas in the form of high-velocity Hiclouds (HVC; Oort 1970). Formally, these are neutral gas structures at a Galactic latitude $|b|>30^{\circ}$ with kinematic properties not consistent with the overall Galactic rotation (Wakker 2001). We now recognize that many of these make up the Magellanic Stream (MS; Dieter 1971; Wannier \& Wrixon 1972; Mathewson et al. 1974), roughly $2 \times 10^{9} M_{\odot}[d / 55 \mathrm{kpc}]^{2}$ of gas (Fox et al. 2014) that has been stripped from the Magellanic Clouds (MCs), two dwarf galaxies in orbit around the Galaxy at a mean distance $d \approx 55 \mathrm{kpc}$ (Walker 2012; Graczyk et al. 2014).

The MS is ideal to study the environment of the Galaxy. Radio (H $21 \mathrm{~cm}$ ) surveys show that the Stream extends for $200^{\circ}$ across the southern Galactic hemisphere (Nidever et al. 2010), and absorption line measurements toward distant quasars indicate a cross-section of roughly one quarter of the whole sky (Fox et al. 2014). Dynamical models (Besla et al. 2007; Guglielmo et al. 2014) constrained by accurate measurements of the proper motions of the MCs (Kallivayalil et al. 2013) agree that they move on a highly eccentric orbit, and that the MS spans a wide range in Galactocentric distance, from its source in the MCs system at roughly $55 \mathrm{kpc}$ to 80 $-150 \mathrm{kpc}$ above the South Galactic Pole (SGP) all the way to the tip of the tail.

Given its relative proximity, the MS has been observed across the electromagnetic spectrum. Beyond $\mathrm{HI}$, it has been detected in molecular (Richter et al. 2001) and ionized ( $\mathrm{Lu}$ et al. 1994; Sembach et al. 2003; Fox et al. 2005) gas. A shadowing experiment aimed at measuring the coronal soft $\mathrm{X}$-ray emission discovered that the emission is enhanced in the direction of the MS (Bregman et al. 2009). Recombination optical emission $(\mathrm{H} \alpha)$ was detected for the first time by Weiner \& Williams (1996) and later confirmed by others (Reynolds et al. 1998; Putman et al. 2004; Madsen 2012). But despite repeated attempts, to date, no stars have been discovered at any location along the Stream (e.g., Ostheimer et al. 1997). The data obtained by the recently completed Hubble Space Telescope (HST) Cosmic Origins Spectrograph (COS) UV absorption survey of the MS (Fox et al. 2013, 2014; Richter et al. 2013) indicate that the Stream is dominated by ionized gas, as was first proposed by Bland-Hawthorn et al. (2007). These data collectively suggest the existence of a strong interaction between the Stream gas and the hot halo (or corona; Spitzer 1956) of the Galaxy.

Radiative hydrodynamic models (the "shock cascade"; Bland-Hawthorn et al. 2007) indicate that the MS-halo interaction may be strong enough to explain the observed disruption of the Stream (Nidever et al. 2010) and its high ionization fraction. At the same time, the presence of coherent and strong enough magnetic fields (Putman et al. 1998) may stabilize the gas against severe ablation and provide thermal insulation to inhibit total evaporation of the neutral clouds (McClure-Griffiths et al. 2010). Whether such a shielding mechanism is operating all along the Stream is currently unknown. But without it, the Stream is likely to evaporate and mass-load the Galactic halo with a substantial amount of baryons.

Therefore, if we are to understand the complex environment of galaxies and how gas settles into galaxies (Heitsch \& Putman 2009), we need to first explain the observed properties of the MS within the framework of a multiphase hydrodynamical model. Elucidating the mechanism behind the bright spots of $\mathrm{H} \alpha$ emission observed along the MS has proven to be 
particularly challenging (see Bland-Hawthorn et al. 2007). To date, there have been two competing models (see also Konz et al. 2001): (i) the slow shock cascade discussed above; (ii) a new interpretation invoking a powerful flare of UV radiation from the Galactic Centre (GC) powered by the accretion of material onto the central black hole in Sgr A* (Bland-Hawthorn et al. 2013).

The GC flare model is inspired by two important circumstances: (1) the discovery of the $\gamma$-ray emitting bubbles discovered by the Fermi satellite extending roughly $50^{\circ}$ $(10 \mathrm{kpc})$ from the GC (Su et al. 2010); (2) the observation that the brightest optical emission along the Stream is confined to a cone with half-angle $\theta_{1 / 2} \approx 25^{\circ}$ roughly centered on the SGP (Madsen 2012). The GC flare model has found support from the timescales and energy budget required to ionize the Stream, which are consistent with the results from jet-driven numerical models of the Fermi bubbles (Guo \& Mathews 2012). More recently, Fox et al. (2014) have discovered that the ionization levels over the SGP require an energetically harder ionizing spectrum than elsewhere along the Stream, with the exception of a localized region near the Large Magellanic Cloud (LMC).

The shock cascade model, on the other hand, explains the observation that the brightest $\mathrm{H} \alpha$ detections lie at the leading edges of the $\mathrm{H}$ iclouds that make up the MS (Weiner et al. 2002). However, this model may fail to produce the observed emission levels if the distance to the Stream at the SGP significantly exceeds the traditional view of $d=55 \mathrm{kpc}$, as indicated by most orbit calculations for the MCs over the past five years (Besla et al. 2012; Guglielmo et al. 2014). The shock cascade model is strongly dependent on the density structure of the Galactic hot halo, and it assumes that the coronal density smoothly declines with Galactocentric distance as $\propto r^{-2}$. But it now appears that both of these assumptions may be false, justifying our efforts to revisit the shock cascade model.

The goal of this study is to investigate the strength of the recombination $(\mathrm{H} \alpha)$ emission produced by the interaction of the MS gas with the Galactic corona exploring a range of Galactocentric distances and different halo parameters, i.e., adopting different density profiles and temperatures of the gas sitting at rest in a fixed dark-matter (DM) potential. Note that throughout the paper we assume a flat, dark-energy- and matter (baryonic and cold dark-matter; CDM) dominated universe, and a cosmology defined by the set of parameters (relevant to this work) $h=0.7, \Omega_{m}=0.3$, and $\Omega_{\Lambda}=0.7$.

\section{A MODEL OF THE GALACTIC HALO}

The density and temperature structure of the Galactic corona are largely determined by the underlying gravitational potential. The potential, in turn, is determined by the three main components of the Galaxy: the stellar bulge, the stellar and gaseous disk, and the DM halo. Given the mass of the bulge $\left(\sim 10^{10} M_{\odot}\right)$ and the disk $\left(\sim 10^{11} M_{\odot}\right.$; Kafle et al. 2014$)$, and their size, these components are expected to dominate the Galactic potential only at $r \lesssim 5 \mathrm{kpc}$ and at $r \lesssim 15 \mathrm{kpc}$, respectively. In other words, with exception of the inner $\sim 5 \mathrm{kpc}$ (Wegg et al. 2015), the DM halo dominates the Galactic potential at all distances, with a similar contribution from the disk at scales comparable to its length. The distance to the nearest point of the MS is believed to be $d \approx 55 \mathrm{kpc}$, which is the average distance to the LMC ( $\approx 50 \mathrm{kpc}$; Walker 2012$)$, and the SMC ( $\approx 60 \mathrm{kpc}$; Graczyk et al. 2014). Consequently, in modeling the interaction of the MS with the Galactic corona, it is safe to ignore the contributions to the potential from the bulge and the disk, and to focus instead on the DM halo only.

\subsection{The Galactic DM Halo}

We model the DM halo of the Galaxy assuming it is well described by a single-component isothermal sphere. We opt for such a model given its solid physical foundation, with properties that can be derived from first principles starting from a few basic assumptions (see e.g., Binney \& Tremaine 2008).

The scale-free potential, $W$, and the scale-free density, $y$, of an isotropic isothermal (DM) sphere are defined by (King 1966):

$$
\begin{gathered}
\frac{1}{x^{2}} \frac{d}{d x}\left(x^{2} \frac{d W}{d x}\right)=-9 y_{\mathrm{ISO}}(x) \\
y_{\mathrm{ISO}}(x)=\exp [W(x)]
\end{gathered}
$$

where $x$ is a scale-free coordinate.

This system of equations has no analytic solution, but it can be integrated numerically to values $x \ll 1$ to (nearly) arbitrary precision. Appropriate boundary conditions are, for example, the requirement that both the potential and the force vanish at the origin, i.e., $W(0) \equiv 0$ and $W^{\prime}(0) \equiv 0$, respectively, which implies $y_{\mathrm{ISO}}(0)=1$. Note that $W(x) \leqslant 0$ for $x \geqslant 0$.

The connection between the scale-free quantities and their physical counterparts is given by

$$
r=r_{\mathrm{c}} x ; \quad \rho(r)=\rho_{\mathrm{c}} y_{\mathrm{ISO}}(x) ; \quad \psi(r)=\sigma^{2} W(x) .
$$

Here, $\rho_{\mathrm{c}}, r_{\mathrm{c}}$, and $\sigma$ are the central density, the core radius, and the constant velocity dispersion, respectively. A solution corresponding to a particular physical system is obtained by fixing two of these three parameters (or any other two independent physical quantities of the system, for that matter); the third parameter is tied to the other two through the relation $r_{\mathrm{c}}^{2}=9 \sigma^{2} / 4 \pi G \rho_{\mathrm{c}}(\mathrm{King} 1966)$.

Alternatives to the isothermal sphere as a viable choice to describe a self-gravitating system of collisionless, DM particles, i.e., a DM halo include the Navarro et al. (1996, NFW) model,

$$
y_{\mathrm{NFW}}(r)=\left(r / r_{\mathrm{s}}\right)^{-1}\left(1+r / r_{\mathrm{s}}\right)^{-2},
$$

where $r_{\mathrm{s}}$ is a characteristic scale length; and the Einasto (1965) profile,

$$
y_{\mathrm{EIN}}(r)=\exp \left\{-\frac{2}{\alpha}\left[\left(\frac{r}{r_{\mathrm{s}}}\right)^{\alpha}-1\right]\right\},
$$

where $\alpha$ is a free parameter. Regardless of the model adopted, if the density profile $\rho(r)$ is known, a general formalism can be applied which allows us to calculate scale-free quantities (q.v. Sternberg et al. 2002) on a case-by-case basis to describe a particular physical system.

In order to specify our isothermal DM halo, and to compare its properties to the NFW and Einasto models, we proceed as follows. Based on the results of Kafle et al. (2014), who assume the dark halo of the Galaxy to be of NFW type, we fix the virial mass $M_{\mathrm{vir}}$ and the concentration $x_{\mathrm{vir}} \equiv r_{\mathrm{vir}} / r_{\mathrm{c}}$ of the NFW dark 
Table 1

DM Halo Properties

\begin{tabular}{lccc}
\hline \hline & Isothermal & Einasto $^{\mathrm{a}}$ & NFW \\
\hline$M_{\text {vir }}\left(10^{12} M_{\odot}\right)$ & $\mathbf{1 . 0 0}$ & $\mathbf{1 . 0 0}$ & $\mathbf{1 . 0 0}$ \\
$r_{\text {vir }}(\mathrm{kpc})$ & 259 & 259 & 259 \\
$v_{\text {vir }}\left(\mathrm{km} \mathrm{s}^{-1}\right)$ & 129 & 129 & 129 \\
$x_{\text {vir }}$ & 433 & 13.8 & $\mathbf{1 5 . 0}$ \\
$\psi_{\text {vir }}\left(10^{15} \mathrm{~cm}^{2} \mathrm{~s}^{-2}\right)$ & $-\mathbf{1 . 1 1}$ & $-\mathbf{1 . 1 1}$ & -1.11 \\
$r_{\mathrm{S}}\left(\mathrm{kpc}^{\mathrm{c}}\right.$ & 0.60 & 18.8 & 17.3 \\
$v_{\mathrm{S}}\left(\mathrm{km} \mathrm{s}^{-1}\right)^{\mathrm{d}}$ & 90.1 & 82.4 & 104 \\
$M_{\mathrm{h}}\left(\leqslant r_{\text {vir }}\right)\left(10^{10} M_{\odot}\right)^{\mathrm{e}}$ & 1.41 & 3.03 & 1.32 \\
$T_{\mathrm{DM}}\left(10^{5} \mathrm{~K}\right)^{\mathrm{f}}$ & 5.81 & 4.85 & 7.73 \\
\hline
\end{tabular}

Notes. Fixed parameter values are shown in bold face; derived values, in normal font face. See the text for details.

${ }^{\mathrm{a}}$ We adopt $\alpha=0.18$ (Gao et al. 2008).

b Gives the value of the "concentration." The value $x_{\text {vir }}=15$ is from Kafle et al. (2014).

${ }^{\mathrm{c}}$ Corresponds to the scale radius for the Einasto and NFW profiles, and to the core radius $r_{\mathrm{c}}$ for the isothermal sphere.

${ }^{\mathrm{d}}$ Corresponds to the scale velocity for the Einasto and NFW profiles, and to the velocity dispersion $\sigma$ for the isothermal sphere.

e Obtained from Equation (10), assuming $\tau \equiv 1$ and $\mu=0.59$.

${ }^{\mathrm{f}}$ Obtained from Equation (7), assuming $\mu=0.59$.

halo to $M_{\mathrm{vir}}=10^{12} M_{\odot}$, and $x_{\mathrm{vir}}=15$, respectively. Our adopted value for the virial mass implies ${ }^{3} r_{\mathrm{vir}} \approx 260 \mathrm{kpc}$ and $v_{\mathrm{vir}} \equiv\left(\begin{array}{ll}G & M_{\mathrm{vir}} / r_{\mathrm{vir}}\end{array}\right)^{1 / 2} \approx 130 \mathrm{~km} \mathrm{~s}^{-1}$, respectively. Then we calculate the scale parameters for the isothermal and the Einasto DM halos by requiring that the virial mass and the physical potential at the virial radius in each case match the corresponding values for the NFW dark halo. Table 1 summarizes our assumed values (in bold font face) and lists the derived values of the relevant scaling parameters for each of the three halo models. The potential and corresponding mass for each model are shown in Figure 1. As can be seen, the relevant properties of an isothermal DM halo are very similar to the NFW and Einasto models. In particular, we note that the virial temperature is comparable across models, which is of relevance for the discussion in later sections.

\subsection{The Galactic Corona}

It has long been known that the potential well of the Galaxy is filled with a diffuse, hot, gaseous component (the corona; Spitzer 1956). However, the origin of this gas, its thermodynamic state, its physical properties, its extension and hence its total mass, are still unknown. A recent attempt to constrain the density structure of the corona implies a total gas mass of the Galaxy around $\sim 10^{10} M_{\odot}$ within $r_{\text {vir }}$ (Miller \& Bregman 2013, 2015), which is consistent with other estimates (e.g., Gatto et al. 2013). This important result relies on the assumptions that the corona is smooth, in collisional ionization equilibrium, and isothermal, with a temperature of $2 \times 10^{6} \mathrm{~K}$. While the gaseous halo of the Galaxy is most likely not smooth

\footnotetext{
3 The viral mass and the virial radius are linked to one another through the relation

$$
r_{\mathrm{vir}}=\left(\frac{3 M_{\mathrm{vir}}}{4 \pi \Delta_{\mathrm{c}} \bar{\rho}_{m}}\right)^{1 / 3} .
$$

We adopt a value for the cosmic mean matter density $\bar{\rho}_{m} \approx 2.76 \times 10^{-30} \mathrm{~g} \mathrm{~cm}^{-3}$ and $\Delta_{\mathrm{c}} \approx 337$. Note that we define the virial radius at $z=0$ (cf. Shull 2014).
}

nor strictly isothermal, the mean temperature of the gas inferred from its associated X-ray emission appear remarkably uniform across the sky (Henley \& Shelton 2013, 2014).

Given this circumstance, we model the hot halo of the Galaxy as a single-phase, smooth, spherically symmetric component consisting of an ideal gas at a constant temperature $T_{\mathrm{h}}$, in hydrostatic equilibrium with the DM potential $\psi$ of an isothermal sphere. We further assume that the self-gravity of the gas is negligible, which is justified in the case of the Galaxy given that the inferred gas mass of the hot halo is on the order of $10^{-2}$ the mass of the DM halo (Suto et al. 1998). Under these assumptions, the total particle density of the corona is given by $n(r)=n_{0} \exp \left[\psi(r) / a^{2}\right]$. Here, $a^{2}=k T_{\mathrm{h}} / \mu m_{u}$ is the isothermal sound speed, $\mu$ is the mean molecular weight, $m_{u}$ is the atomic mass unit, and $k$ is Boltzmann's constant.

The virial "temperature" of the dark matter halo follows from the equivalence $a^{2} \equiv \sigma^{2}$,

$$
T_{\mathrm{DM}} \equiv \frac{\mu m_{u}}{k} \sigma^{2}
$$

It worth emphasizing that the above is is merely an equivalent temperature, as $\mu m_{u}$ is not literally the DM particle mass (which is currently unknown). Table 1 lists the virial temperatures of the different models.

It is straightforward to show that under these assumptions the distribution of the hot gas in the potential is effectively governed by the thermal ratio (Cavaliere \& FuscoFemiano 1976) ${ }^{4}$

$$
\tau \equiv \frac{T_{\mathrm{DM}}}{T_{\mathrm{h}}}=\sigma^{2} / a^{2},
$$

such that

$$
n(r)=n_{0} \exp \left[\tau W\left(r / r_{\mathrm{c}}\right)\right]
$$

The total gas mass within $r$ for a particular value of $\tau$ follows from the integral of $n$ over the appropriate volume,

$$
M_{\mathrm{h}}(r)=4 \pi \mu m_{u} \int_{0}^{r} n(x) x^{2} d x
$$

Clearly, the density field of gas in hydrostatic equilibrium with a fixed isothermal potential will vary for different gas temperatures. If the gaseous halo is "hotter" than the DM particles, i.e., $\tau<1$, then the density falls off more gently with radius. Values $\tau>1$, on the other, hand reflect the fact that the gas has cooled below the virial temperature of the DM halo, and is hence more concentrated. Large $\tau$ values thus imply lower densities in the outer region of the halo, which in turn leads to weaker hydrodynamic interactions (at a fixed distance).

Since the the virial temperature of our DM halo model is fixed, we control the temperature of the halo gas by varying $\tau$. In the following we consider values of $\tau$ in the range $[0.5,1.5]$ only, and choose the set of values $\tau \in\{1.5,1.0,0.75,0.5\}$ as representative of this range. These values imply gas temperatures $T_{\mathrm{h}} \sim 10^{6} \mathrm{~K}$ (see Table 2), consistent with estimates of the temperature of the Galactic hot halo (Snowden et al. 2000). We ignore values of $\tau<0.5$, since these yield overly shallow density profiles, which are inconsistent with observations (see Figure 3). Similarly, we ignore values of $\tau>1.5$ because these

\footnotetext{
4 Note that the designation of thermal ratio by the Greek letter $\beta$ is widespread in the literature. Here, we adopt its original designation.
} 

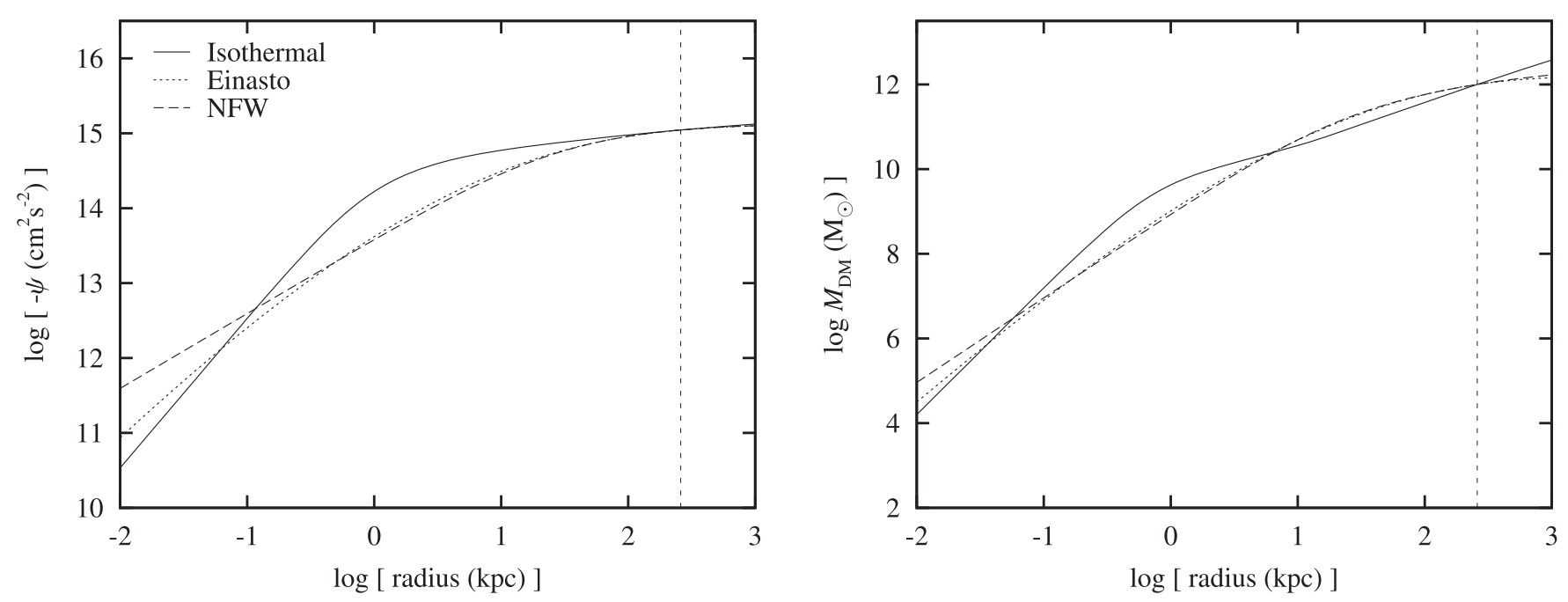

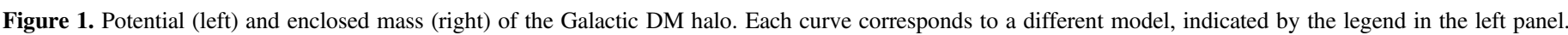

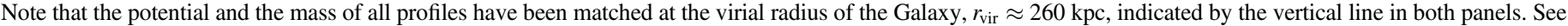
also Table 1 .

Table 2

Isothermal Gaseous Halo Properties

\begin{tabular}{lcc}
\hline \hline$\tau$ & $T_{\mathrm{h}}\left(10^{6} \mathrm{~K}\right)$ & $M_{\mathrm{h}}\left(r_{\mathrm{vir}}\right)\left(10^{10} M_{\odot}\right)$ \\
\hline 0.5 & 1.16 & 3.36 \\
0.75 & 0.77 & 2.06 \\
1.0 & 0.58 & 1.41 \\
1.5 & 0.39 & 2.33 \\
\hline
\end{tabular}

imply gas temperature which are too low compared to observations (see Section 3.2).

We compare in Figure 2 the density profiles of gas at rest in different DM potentials, all scaled to a fiducial value $n=2 \times 10^{-4} \mathrm{~cm}^{-3}$ at $55 \mathrm{kpc}$ (see below and Section 3.2). For a given $\tau$, the gas density profile within an NFW DM potential (as defined by the respective parameters in Table 1) is steeper compared to the isothermal DM potential, but shallower than the Einasto DM halo. The difference in the density profile across the models reflects the difference in the potential in the radial range of interest. In contrast, the gas masses enclosed within $r_{\text {vir }}$ for all three models and a given $\tau$ are comparable (with exception of the mass for the Einasto model with $\tau=0.5$ which is well above $\left.10^{11} M_{\odot}\right)$. Therefore, of all three models at a fixed $\tau$, the isothermal sphere halo leads to the highest gas density at any given distance beyond $55 \mathrm{kpc}$ for roughly the same gas mass, and hence to the strongest hydrodynamic interaction in the outer halo.

The different density profiles of gas sitting in an isothermal DM halo for our adopted values of $\tau$ (Equation (9)), scaled to $n=2 \times 10^{-4} \mathrm{~cm}^{-3}$ at $55 \mathrm{kpc}$, are shown in the top-left panel of Figure 3. For comparison, we include there a set of values of the halo density at various Galactocentric distances obtained from observations using a variety of methods (see Section 3.2). It is reassuring that all our models are fairly consistent with these measurements.

The top-right panel of Figure 3 displays the mass enclosed within a given radius (Equation (10)) for each of the models shown in the top-left panel. As a consistency check, we compare the model masses to the upper limit on the Galactic baryion budget within $r_{\text {vir }}$ set by the universal mean baryon-to- total mass ratio $f_{b} \equiv \Omega_{b} / \Omega_{m}$. The most recent estimates of the baryon and cold dark matter mass densities $\Omega_{b} h^{2}=0.02205 \pm$ 0.00028 and $\Omega_{\mathrm{c}} h^{2}=0.1199 \pm 0.0027$ (Planck Collaboration 2014) imply $f_{b} \approx 0.16$. This value, together with the total mass of the Galaxy $\left(M_{\mathrm{tot}} \sim 10^{12} M_{\odot}\right)$ allows for a maximum gas mass of the Galaxy within $r_{\mathrm{vir}}$ below $10^{11} M_{\odot}$. Clearly, all our models result in masses within $r_{\text {vir }}$ which are below this limit, indicated by the gray hatched area in the right panel of Figure 3. Note that a density profile shallower than the $\tau=0.5$ model, or a value of $n(55 \mathrm{kpc})$ significantly higher than our fiducial value, would result in a gas mass largely inconsistent with these constraints. We include in this figure the range of hot halo masses inferred from observations by Miller \& Bregman (2015), comparable to the masses estimated by others (e.g., Gatto et al. 2013), and the somewhat lower values inferred by Salem et al. (2015). Note that these estimates are all directly comparable to our model results since in all cases a smooth, monotonically decreasing density profile has been assumed. All our models, with the exception of the $\tau=0.5$ model, predict masses within $r_{\text {vir }}$ that are consistent with the inferred mass.

Thus, the model $\tau=0.5$ appears to be marginally consistent both with the mean density of the Galactic corona at large distances, and with the constraint on the total gas mass of the Galaxy. The model $\tau=1.5$, although compatible with these constraints, appears too concentrated to be a plausible description of the Galaxy's halo. In contrast, the models $\tau=0.75$ and $\tau=1$ both display the best performance in terms of both the density profile and the gas mass enclosed within the virial radius of the Galaxy, although the former model yields a slightly hotter and more massive gas halo. In addition, these models reproduce by construction the relevant properties of the Galactic DM halo. Thus, we consider that these models in particular provide a fully self-consistent and well founded description of the Galactic hot halo, despite its idealized nature.

\section{NUMERICAL EXPERIMENT}

We simulate the passage of a stream of gas emulating the MS in its orbit through the Galactic hot halo by means of a three-dimensional (3D) "wind-tunnel experiment," expanding on the work by Bland-Hawthorn et al. (2007). In brief, we 

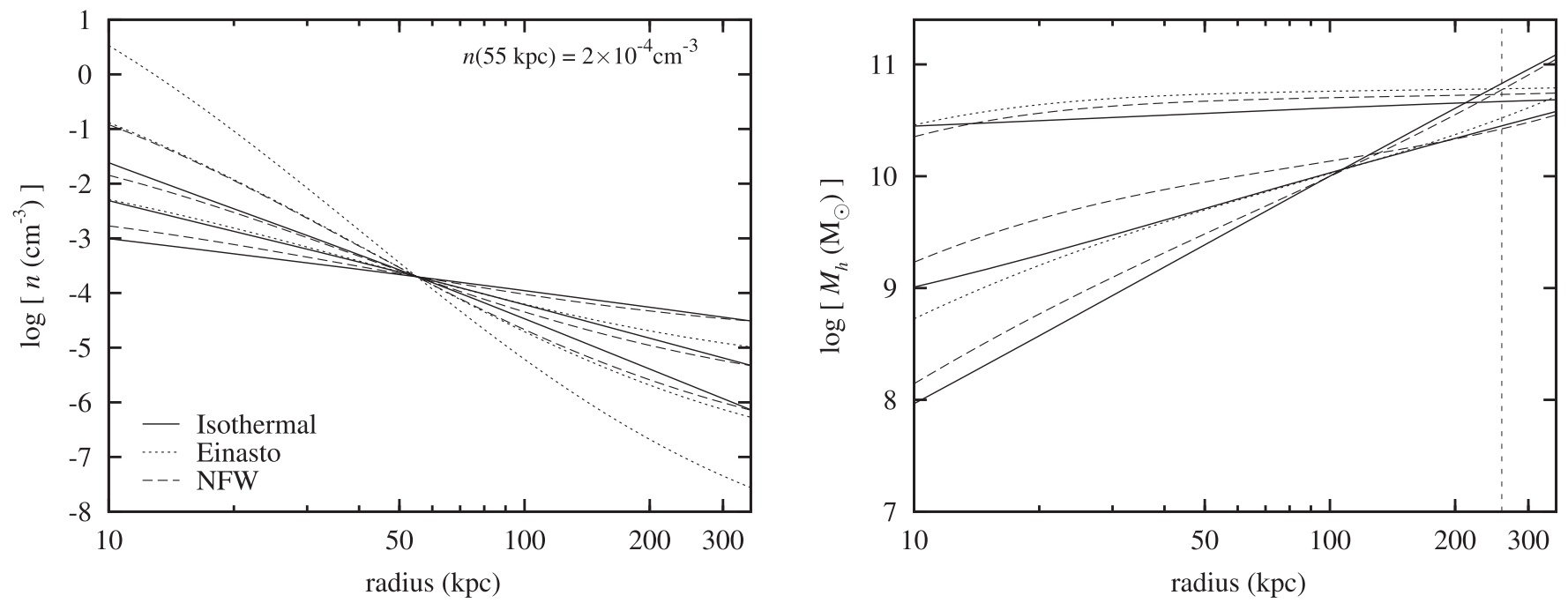

Figure 2. Left: density profile model (Equation (9)) adopting different DM halo models, all normalized to $n(55 \mathrm{kpc})=2 \times 10^{-4} \mathrm{~cm}^{-3}$. For a given halo, the curves correspond, from top to bottom, to values of $\tau=1.5,1.0$, and 0.5 . Right: gas mass enclosed within a given radius (Equation (10)) for each of the density profiles shown in the left panel. Note that the order of the curves is inverted with respect to the order on the left panel, i.e., for a given halo model, the lowest curve corresponds to $\tau=1.5$, etc. The vertical line indicates the virial radius of the Galaxy, $r_{\mathrm{vir}} \approx 260 \mathrm{kpc}$. Note that the enclosed mass for the Einasto model with $\tau=0.5$ is well above $10^{11} M_{\odot}$ and is not shown.

place a warm and essentially neutral, fractal gas cloud at a distance $r_{\mathrm{MS}}$ initially at rest with respect to the computational volume, and exposed it to a hot wind at a constant temperature $T_{\mathrm{h}}$ (for a fixed $\tau$ ) and constant density $n$ (for a fixed $\tau$ and $r_{\mathrm{MS}}$ ) flowing with velocity $\boldsymbol{v}_{\mathrm{h}}$ under a fixed impact angle $\vartheta$ with respect to the gas cloud (see below). The warm gas is assumed to be initially in a state of pseudo-equilibrium with the hot gas, defined by the mean cloud-to-halo density ratio (or overdensity) $\eta \equiv \rho_{w} / \rho_{\mathrm{h}}$, and the mean cloud-to-halo pressure ratio $\xi \equiv P_{w} / P_{\mathrm{h}}$ (see Equation (11)).

\subsection{Code}

We choose for our experiment the high-resolution, multiphase, shock-capturing hydrodynamic grid-based code FYRIS ALPHA (Sutherland 2010), especially developed for astrophysical applications. The code solves the fluid dynamic equations in one, two, and three dimensions as required. It has been shown to be fast, robust and accurate when compared to similar codes, and it performs well when subject to a standard suite of test cases as developed by Liska \& Wendroff (2003). A unique feature of the FYRIS ALPHA code is that it includes nonequilibrium cooling through time-dependent ionization calculations. In addition, the code allows for the use of a variable equation of state through a variable adiabatic index $\gamma$ and/or a variable mean molecular weight $\mu$. These features are essential due to the large difference in the relevant timescales which determine the physical state of multi-phase gas, as well as the large range of densities encountered in these type of simulations.

\subsection{Observational Constraints, Initial Conditions, and Set-up}

The proper motion of the LMC has recently been measured using HST data, yielding an orbital velocity $v_{\mathrm{LMC}}=321 \pm$ $24 \mathrm{~km} \mathrm{~s}^{-1}$ (Kallivayalil et al. 2013). We adopt the high-end value and set the speed of the hot wind to $v_{\mathrm{h}}=350 \mathrm{~km} \mathrm{~s}^{-1}$. Also, we adopt a value for the impact angle $\vartheta=24^{\circ}$, such that the (shear) velocity of the hot wind is given by $v_{\mathrm{h}}=\left(v_{\mathrm{h}} \cos \vartheta, v_{\mathrm{h}} \sin \vartheta, 0\right)=(320,141,0) \mathrm{km} \mathrm{s}^{-1}$.
Note that our results are fairly insensitive to the adopted value of $\vartheta$, as long as $0 \ll \vartheta \ll \pi / 2$, which is supported by the belief that the orbit of the MS is likely neither radial nor tangential with respect to the gaseous Galactic halo. We will assess the impact of this plausible, albeit arbitrary, choice on our results when dealing with virtual observations in Section 4 below.

The Stream's mean metallicity away from the MCs is now well constrained to $Z \approx 0.1 Z_{\odot}$ (Richter et al. 2013; Fox et al. 2014). In contrast, the metallicity of the halo gas is still uncertain, although cosmological simulations (Rasmussen et al. 2009) and pulsar dispersion measures toward the LMC (Miller \& Bregman 2015) both suggest that it is likely in the range $Z \sim 0.1 Z_{\odot}-0.3 Z_{\odot}$ far away from the disk. We choose a value for the metallicity of the halo of $Z=0.1 Z_{\odot}$, which is consistent with the mean value observed in external galaxies similar to the MW (NGC 891; Hodges-Kluck \& Bregman 2013).

Only gas clouds that are not overly dense and which have low pressure support with respect to the ambient medium will be disrupted in realistic timescales of $\sim 100 \mathrm{Myr}$ (BlandHawthorn 2009). We adopt $\eta=100$ and $\xi=0.1$. With these parameters fixed, the initial temperature of the warm gas phase is set by the temperature of the hot gas phase through

$$
T_{w}=\left(\frac{\mu_{w}}{\mu_{\mathrm{h}}}\right)\left(\frac{\xi}{\eta}\right) T_{\mathrm{h}}
$$

where it should be noted that the mean molecular weight will be generally different in each phase.

A key parameter of the models is the normalization of the density profile at the canonical distance of the Stream above the SGP $(d=55 \mathrm{kpc})$. Although still uncertain, different lines of evidence indicate that it is likely in the range of $10^{-5} \mathrm{~cm}^{-3}$ $-10^{-3} \mathrm{~cm}^{-3}$ at $20 \mathrm{kpc} \lesssim r \lesssim 100 \mathrm{kpc}$. For example, Blitz \& Robishaw (2000) estimate a lower limit on the mean halo density of $n \approx 2.4 \times 10^{-5} \mathrm{~cm}^{-3}$ out to $d \leqslant 250 \mathrm{kpc}$ based on the assumption that the gas-poor dwarf spheroidals orbiting the Galaxy have been stripped from their gas by the ram-pressure exerted by the hot halo. Along the same line, and combining 

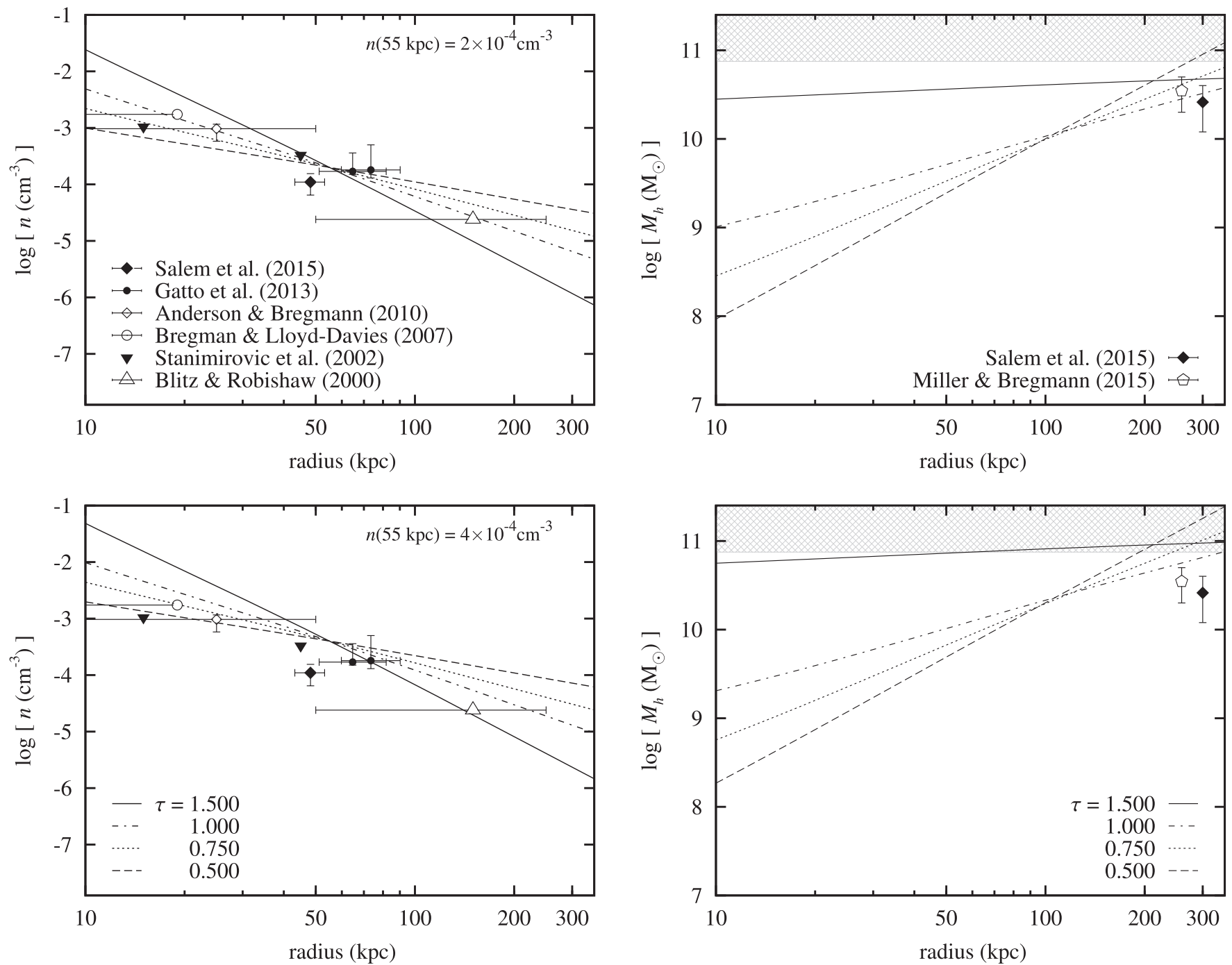

Figure 3. Left: density profile model (Equation (9)) for different values of $\tau$. Also shown are a set of halo density measurements at a range of Galactocentric distances. Right: gas mass enclosed within a given radius (Equation (10)) for each of the density profiles shown in the left panel. The data points indicate the range of Galactic halo gas masses within $r=300 \mathrm{kpc}$ inferred from observations. The gray hatched area on the top of the panel indicates the range of gas masses which are too large to be consistent with the (mean) cosmic baryon-to-total mass ratio $f_{b} \approx 0.16$ (see the text for details). Note that the top panels correspond to models that have been scaled to a fiducial value $n(55 \mathrm{kpc})=2 \times 10^{-4} \mathrm{~cm}^{-3}$. The bottom panels show the corresponding results adopting $n(55 \mathrm{kpc})=4 \times 10^{-4} \mathrm{~cm}^{-3}$ instead. These are discussed in Section 4.4.

observations with (2D) hydrodynamic simulations, Gatto et al. (2013) have inferred a range of halo densities $n \approx(1-4) \times$ $10^{-4} \mathrm{~cm}^{-3}$ at $50 \mathrm{kpc}<d<100 \mathrm{kpc}$. Stanimirović et al. (2002) have found that the gas clouds at the tail of the MS are likely in pressure equilibrium with the hot halo, and using this they have put an upper limit on the halo density of $10^{-3} \mathrm{~cm}^{-3}$ and $3 \times 10^{-4} \mathrm{~cm}^{-3}$ at a distance $z=15 \mathrm{kpc}$ and $z=45 \mathrm{kpc}$ from the Galactic plane, respectively. Anderson \& Bregman (2010) infer a range for the mean halo density of $n \approx(6-10) \times 10^{-4}$ out to the LMC ( $d \approx 50 \mathrm{kpc})$ based on dispersion measures of LMC pulsars. However, the stripping of the LMC's disk requires a somewhat lower value of $n(48.2 \pm 3 \mathrm{kpc})=$ $(1.1 \pm 0.44) \times 10^{-4} \mathrm{~cm}^{-3}$ (Salem et al. 2015).

Based on these results, we adopt a fiducial value $n$ $(55 \mathrm{kpc})=2 \times 10^{-4} \mathrm{~cm}^{-3}$, consistent with Bland-Hawthorn et al. (2007). As shown in Figure 3, this choice leads to models for the Galactic corona that largely agree with the results from observations over a broad range in distances. In this respect, we consider both $n(55 \mathrm{kpc})$ and $\tau$ to be well constrained by observation. Note that the models are completely defined by the value of $\tau$, given that all the other parameters are either fixed or they depend on $\tau$ (Table 3).

We run all simulations in a rectangular box of comoving size $18 \times 9 \times 9 \mathrm{kpc}^{3}$, using a fixed grid composed of $432 \times 216 \times 216$ cells. These settings imply a spatial resolution of $\delta x=(9 / 216) \mathrm{kpc} \approx 42 \mathrm{pc}$. The fragment of gas representing the MS is initially constrained to a cylinder $18 \mathrm{kpc}$ in length and $2 \mathrm{kpc}$ in diameter, and whose axis of symmetry runs parallel to the $x$ axis of the coordinate system defined by the box. The impact angle is defined with respect to the $x$-axis of this cylinder. In this setup, the $x$-axis coincides with the Magellanic longitude, $l_{\mathrm{M}}$, whereas any of $y$ or $z$ run along the Magellanic latitude, $b_{\mathrm{M}}$ (Wakker 2001; Nidever et al. 2008). The simulated MS consists of an $\mathrm{H}$ Igas distribution initially at temperature $T_{w} \sim 10^{3} \mathrm{~K}$ (see Equation (11)); a mean initial hydrogen particle density $n \sim$ $10^{-3} \mathrm{~cm}^{-3}$; and a total neutral gas mass $M_{w}(\mathrm{HI}) \sim 10^{7} M_{\odot}$. The initial warm gas density field corresponds to the density of 
Table 3

Relevant Simulation Parameters/Initial Conditions

\begin{tabular}{lcl}
\hline \hline Parameter & Value & Remarks \\
\hline$\left(n_{x}, n_{y}, n_{z}\right)$ & $(432,216,216)$ & Grid dimensions \\
$(x, y, z)(\mathrm{kpc})$ & $(18,9,9)$ & Physical dimensions \\
$\delta x(\mathrm{pc})$ & 42 & Spatial resolution (approximate) \\
$T_{\mathrm{h}}(\mathrm{K})$ & $10^{6}$ & Halo gas temperature \\
$T_{w}(\mathrm{~K})$ & $10^{3}$ & Initial Stream gas temperature $^{\mathrm{b}}$ \\
$M_{w}(\mathrm{H})\left(M_{\odot}\right)$ & $10^{7}$ & Initial Stream neutral gas mass \\
$\eta$ & 100 & Initial ratio of cloud:halo density \\
$\xi$ & 0.1 & Initial ratio of cloud:halo pressure \\
$n(55 \mathrm{kpc})\left(\mathrm{cm}^{-3}\right)$ & $2 \times 10^{-4}$ & Total particle density at $55 \mathrm{kpc}$ \\
$Z_{\mathrm{h}}\left(Z_{\odot}\right)$ & 0.1 & Halo gas metallicity \\
$Z_{w}\left(Z_{\odot}\right)$ & 0.1 & Stream's metallicity \\
$X$ & 0.7154 & Hydrogen mass fraction \\
$Y$ & 0.2703 & Helium mass fraction \\
$\vartheta\left({ }^{\circ}\right)$ & 24 & Impact angle \\
$\Delta v\left(\mathrm{~km} \mathrm{~s}^{-1}\right)$ & 200 & Velocity range of emission spectra \\
& & with pixel size $\delta v=2$ km s \\
\hline
\end{tabular}

Notes.

a Approximate. See Table 2.

b Approximate. The exact value will vary by small factors depending on $T_{\mathrm{h}}$ (see Equation (11))

a fractal medium described by a Kolmogorov turbulent power spectrum $P(k) \propto k^{-5 / 3}$, with a minimum wavenumber $k_{\min }=8$ (relative to the grid) corresponding to a spatial scale of $2.25 \mathrm{kpc}$ (q.v. Sutherland \& Bicknell 2007), comparable to the typical size of clouds in the Stream. The Higas cloud is assumed to be at a fixed distance $r_{\mathrm{MS}}$ from the GC in the direction of the SGP. The temperature $T_{\mathrm{h}}$ and the density $n$ of the hot wind are set according to the value of $\tau$ and the distance $r_{\mathrm{MS}}$ as given by Equations (7) and (9), respectively.

For each $\tau \in\{0.5,0.75,1.0,1.5\}$, we consider a set of Galactocentric distances $r_{\mathrm{MS}} \in\{55,75,100,125,150\} \mathrm{kpc}$ which together span the range of plausible orbits of the MS above the SGP (Guglielmo et al. 2014). This yields 20 models. Each model is run for a total (simulation) time of $320 \mathrm{Myr}$, starting from $t_{\text {sim }}=0 \mathrm{Myr}$, assuming free boundary conditions. The simulation output for a given set of values $\left\{\tau, r_{\mathrm{MS}}, t_{\mathrm{sim}}\right\}-$ in steps of $\Delta t_{\text {sim }}=10 \mathrm{Myr}$ - consists of a series of datacubes containing information about the $\mathrm{H} \mathrm{I}$ - and $\mathrm{H}$ II densities, $n_{\mathrm{H}_{\mathrm{I}}}$ and $n_{\mathrm{H} \text { II }}$, respectively; the gas temperature $T$, and the gas velocity $\boldsymbol{v}=\left(v_{x}, v_{y}, v_{z}\right)$. Using this information, we compute the $\mathrm{H} \alpha$ emission and the $\mathrm{H}$ i column density of each cell, and from these the $\mathrm{H} \alpha$ surface brightness and total $\mathrm{H}$ icolumn density along the sightline for each snapshot.

\subsection{Emission Line Spectra}

We compare the $\mathrm{H} \alpha$ emission of the gas in our simulations to Fabry-Pérot $\mathrm{H} \alpha$ observations along the MS (Bland-Hawthorn et al. 2013, and references therein), and complement these with results on the associated $\mathrm{H}$ i column density measurements.

Given the one-to-one correspondence between the $\mathrm{H}_{\text {I }} 21 \mathrm{~cm}$ emission (i.e., brightness temperature) $T_{\mathrm{B}}$ and the $\mathrm{H}$ i column density of a parcel of optically thin gas (e.g., Dickey \& Lockman 1990), we use the total H i column density along the sightline $\left(N_{\mathrm{H}_{\mathrm{I}}}\right)$ as a proxy for the corresponding $\mathrm{H}_{\mathrm{I}} 21 \mathrm{~cm}$ emission, i.e., we define the $\mathrm{H}_{\text {I }} 21 \mathrm{~cm}$ intensity to be $I_{\mathrm{H}} \equiv N_{\mathrm{H} \text { I }}$.
The $\mathrm{H} \alpha$ emission is computed using

$$
\mu_{\mathrm{H} \alpha}=\mu_{\mathrm{H} \alpha}{ }^{\text {(shock) }}+\mu_{\mathrm{H} \alpha}{ }^{\text {(phot) }} .
$$

The first term accounts for the ionization that results from slow shocks produced by the collision of the trailing cloud gas with the leading gas ablated by the interaction with the hot halo, and is given by

$$
\mu_{\mathrm{H} \alpha}{ }^{\text {(shock) }}=[1+(Y / 4 X)] K_{R} \alpha_{B}^{(\mathrm{H} \alpha)} \int\left(n_{\mathrm{H} \mathrm{II}}\right)^{2} d s,
$$

where $\alpha_{B}^{(\mathrm{H} \alpha)}(T)$ is the effective $\mathrm{H} \alpha$ recombination coefficient (Equation (17)), $K_{R} \approx 1.67 \times 10^{-4} \mathrm{~cm}^{2} \mathrm{~s} \mathrm{mR}^{5}$ and the factor $[1+(Y / 4 X)]$ accounts for the conversion of electron density to $\mathrm{H}$ II particle density. Adopting hydrogen and helium mass fractions $X=0.7154$ and $Y=0.2703$, respectively (Asplund et al. 2009, solar bulk composition) ${ }^{6}$ assuming the gas is fully ionized $\left(n_{\mathrm{H}} \approx n_{\mathrm{H}}\right)$, it follows that $[1+(Y / 4 X)] \approx 1.09 .^{7}$

The second term in Equation (12) accounts for the ionizing effect of the cosmic ultraviolet background radiation (UVB). The $\mathrm{H} \alpha$ emission along the sightline of gas in photoionization equilibrium with the UVB radiation field is

$$
\mu_{\mathrm{H} \alpha}{ }^{\text {(phot) }}=\frac{1}{4 \pi} \Gamma_{\mathrm{H} \mathrm{I}} \int\left(f_{\mathrm{H} \alpha} n_{\mathrm{H} \mathrm{I}}\right) d s,
$$

where $f_{\mathrm{H} \alpha}(T)$ gives the fraction of recombinations that produce an $\mathrm{H} \alpha$ photon, and $f_{\mathrm{H} \alpha}\left(10^{4} \mathrm{~K}\right) \approx 0.45$ (Equation (19)). The $\mathrm{H}$ I recombination rate, $\Gamma_{\mathrm{H}_{\mathrm{I}}}$, is related to the total ionizing photon flux $\Phi_{i}$ through

$$
\Phi_{i}=1.59 \times 10^{4} \text { photon } \mathrm{cm}^{-2} \mathrm{~s}^{-1}\left(\frac{\gamma+3}{4 \gamma}\right)\left(\frac{\Gamma_{\mathrm{H}}}{10^{-13} \mathrm{~s}^{-1}}\right) .
$$

We adopt $\Gamma_{\mathrm{H}_{\mathrm{I}}}=10^{-13} \mathrm{~s}^{-1}$ (appropriate for $z=0$; Weymann et al. 2001) and $\gamma=1.8$ (Shull et al. 1999), corresponding to an ionizing flux $\Phi_{i} \sim 10^{4}$ photons $\mathrm{cm}^{-2} \mathrm{~s}^{-1}$. If instead we used the most recent estimate $\Gamma_{\mathrm{H}}=4.6 \times 10^{-14} \mathrm{~s}^{-1}$ (Shull et al. 2015), the flux would be lower by roughly a factor of 2 .

To mimic radiation transfer effects, we limit the depth (along the sightline in any direction) of the gas ionized by the UVB to a maximum value defined by the condition that the column recombination $\left(n_{e} n_{\mathrm{H} \text { II }} \alpha_{\mathrm{B}}\right)$ equals the incident ionizing photon flux $\left(\Phi_{\mathrm{i}}\right)$. This condition is equivalent to restricting the ionizing effect of the UVB to a column of neutral gas $\sim 10^{17} \mathrm{~cm}^{-2}$ (see Appendix B). The effect of the cosmic UVB is to produce an ionization skin around the cloud featuring an $\mathrm{H} \alpha$ surface brightness at a level of roughly $5 \mathrm{mR}$. Again, if instead we used $\Gamma_{\mathrm{H}}=4.6 \times 10^{-14} \mathrm{~s}^{-1}$, this value would decrease to roughly 2 $\mathrm{mR}$. It is important to mention that this approach is not entirely self-consistent with our simulations because the ionizing effect of the UVB is not included at runtime, and because it assumes photoionization equilibrium. Also, we ignore for the moment the contribution of the Galactic ionizing field, which would produce an additional mean $\mathrm{H} \alpha$ signal of $21 \zeta[d / 55 \mathrm{kpc}]^{-2} \mathrm{mR}$ $(\zeta \approx 2$; Bland-Hawthorn et al. 2013).

\footnotetext{
51 milli-Rayleigh $(\mathrm{mR})$ corresponds to $10^{3} / 4 \pi$ photons $\mathrm{cm}^{-2} \mathrm{~s}^{-1} \mathrm{sr}^{-1}$ (Baker \& Romick 1976), or $2.41 \times 10^{-4} \mathrm{erg} \mathrm{cm}^{-2} \mathrm{~s}^{-1} \mathrm{sr}^{-1}$ at $\mathrm{H} \alpha$.

6 For our adopted metallicity of $10 \%$ the solar value, $Z_{\odot}=0.0142$, the contribution of heavy elements to the electron density can be neglected.

7 We assume that helium is only singly ionized, given that the ionization energy of He II is comparatively high ( $E \approx 54.4 \mathrm{eV}$; Kramida et al. 2014).
} 
To allow for a faithful comparison with observations, we map the simulation data onto observed space by projecting the simulation volume along a given axis, so as to mimic the projection of the observed emission along the Stream onto the plane of the sky. We choose, for convenience, an axis parallel to one side of the simulation box, and perpendicular to the Stream's main axis, i.e., the $y$-axis. However, we will address the potential bias introduced by this choice by comparing the results obtained by projecting along all three orthogonal axes, $x, y$ and $z$ as well.

Each sightline across the projected datacube thus corresponds to a pencil-beam spectrum. $\mathrm{H} \alpha$ and $\mathrm{H}_{\mathrm{I}} 21 \mathrm{~cm}$ pencilbeam spectra along the chosen projection axis are constructed by computing the intensity for each cell in the simulation volume. The $\mathrm{H} \alpha$ intensity and the $\mathrm{H}_{\mathrm{I}} 21 \mathrm{~cm}$ emission intensity observed at velocity $v_{m}$ (in the rest-frame of the $\mathrm{H}_{\mathrm{I}}$ gas at $\left.t_{\text {sim }}=0\right)$ of a parcel of gas at cell $\mathbf{n} \equiv(i, j, k)$ with bulk velocity $v_{\mathrm{n}}$ are, respectively,

$$
\begin{aligned}
& I_{\mathrm{H} \alpha}\left(v_{m}-v_{\mathrm{n}}, \boldsymbol{n}\right)=\mu_{\mathrm{H} \alpha}(\boldsymbol{n}) \phi_{\mathrm{n}}\left(v_{m}-v_{\mathrm{n}}\right), \\
& I_{\mathrm{H} \mathrm{I}}\left(v_{m}-v_{\mathrm{n}}, \boldsymbol{n}\right)=N_{\mathrm{H} \mathrm{I}}(\boldsymbol{n}) \phi_{\mathrm{n}}\left(v_{m}-v_{\mathrm{n}}\right),
\end{aligned}
$$

where the normalized line profile $\phi(v)=\exp \left[-v^{2} / b_{T}^{2}\right]$, with $b_{T}=\left(2 k T_{w} / m_{u}\right)^{1 / 2}$.

We adopt a spectral range in terms of the velocity of $\Delta v=200 \mathrm{~km} \mathrm{~s}^{-1}$ which corresponds to the spectral range provided by the WHAM spectrometer. The velocity scale is given with respect of the initial rest-frame of the $\mathrm{H}$ I gas, such that emission spectrum spans the range $[-100,+100] \mathrm{km} \mathrm{s}^{-1}$, with a pixel size $\delta v=2 \mathrm{~km} \mathrm{~s}^{-1}$.

At each beam position (or "pointing"), we compute the intensity-weighted average of all cells within the beam, resulting in a single spectrum per pointing. We adopt a low resolution beam of $1^{\circ}$ diameter on the sky, identical to the resolution provided by the WHAM spectrometer.

We choose a rectangular (rather than a circular) beam, which allows for a full coverage of the projected image, and which greatly simplifies the scanning procedure. ${ }^{8}$ Given that a beam with a diameter of $2 \theta$ subtends a solid angle $\Omega_{\text {beam }} \approx \pi(\theta / 2)^{2}$ (provided that $\theta \ll 1$ ), the linear size of a square subtending a solid angle $\Omega_{\text {beam }}$ at a distance $r_{\mathrm{MS}}$ relative to the angular dimension of a single cell in our simulation, $\delta \Omega_{V} \approx\left(\delta x / r_{\mathrm{MS}}\right)^{2}$, is roughly $\delta l=(\pi / 4)^{1 / 2}\left(r_{\mathrm{MS}} / \delta x\right) \theta$. The emission within a beam pointing at each velocity $v_{m}$, i.e., the beam spectrum, is then

$$
\bar{I}_{\mathrm{X}}=[\delta l]^{-2} \sum_{[\delta l][\delta l]} I_{\mathrm{X}}
$$

where the sum extends over all cells within $\Omega_{\text {beam, and }}$ $X \in\left\{\mathrm{H}, \mathrm{H}_{\alpha}\right\}$. Here, the notation $[k]$ indicates the largest odd integer smaller than or equal to $k$.

The average of the emission within each beam pointing can effectively be obtained by overlaying a rectangular grid on the projected image with a cell size equal the solid angle subtended by a circular beam of diameter $2 \theta$ at that distance, and computing the arithmetic mean within each new cell. We choose the origin of the matrix to be shifted by half a beam size in each direction $(x y)$ to avoid the uncertainties associated with the simulation volume's boundaries.

\footnotetext{
8 A alternative arrangement consisting of a tightly packed array of circular windows (which conveys equal weight to every pixel within the beam; see e.g. Haffner et al. 2003, their Figure 3) would yield essentially the same results.
}

It is important to emphasize that the pixelation of the simulation volume and the position of the beam pointings with respect to the projected datacube are rather arbitrary. Also, the latter is also generally different for each adopted distance $r_{\mathrm{MS}}$. This results from the fact that for a computational volume with fixed comoving size and fixed grid, and a beam of fixed angular size, an increasing fraction of the gas that represents the Stream will be sampled by the beam with increasing distance. Because of this, and also to avoid a bias in the resulting emission introduced by potentially bright features induced by chance alignments (rather than due to intrinsically bright gas blobs), prior to computing the spatial average within each beam pointing we smooth each $2 \mathrm{D}$ spatial slice at each velocity bin using a circular Gaussian kernel with a full width at half maximum of half the beam size and a total width of (i.e., truncated at) the size of the beam. According to the approach described above, the projected intrinsic $\mathrm{H} \alpha$ emission map from our simulation is smoothed at each given distance using a Gaussian kernel with FWHM of [ $\delta l / 2]$ pixel and a total width of $[\delta l]$ pixel.

Finally, in order to take into account the instrumental line broadening, we convolve each spatially averaged spectrum using a Gaussian kernel $^{9}$ with a FWHM $=12 \mathrm{~km} \mathrm{~s}^{-1}$, which roughly corresponds to the resolution of the WHAM spectrograph (Reynolds et al. 1998). The H $\alpha$ surface brightness and $\mathrm{H}_{\mathrm{I}} 21 \mathrm{~cm}$ emission maps are obtained from the 3D spectral datacube by integrating each spectrum along the velocity coordinate (Equations (16)).

A selected example illustrating the result of the above procedure is shown in Figure 4 (see also Figure 6). As we show below (see Section 4.3; Figure 7), the $\mathrm{H}_{\text {II }}$ to $\mathrm{H}$ I mass ratio in this snapshot roughly matches to the corresponding ratio observed in the MS ( 3; Fox et al. 2014). The effect of the ionizing cosmic UVB is apparent: all the Stream gas is lit up and emitting at a level of $\sim 5 \mathrm{mR}$. But there are brighter spots that are a consequence of the shock cascade, whereby the trailing clouds collide with the material ablated by hydrodynamic instabilities from the leading gas, thus being shock ionized (q.v. Bland-Hawthorn et al. 2007). Interestingly, while the bright $\mathrm{H} \alpha$ spots seem to closely track the high $N_{\mathrm{H}}$ parcels of gas, the converse is not true, with high $N_{\mathrm{H}_{\mathrm{I}}}$ appearing with no correspondent strong $\mathrm{H} \alpha$ emission. However, these differences become less apparent, although they remain, as a result of the beam smearing. The most dramatic effect of the latter is the dilution of the $\mathrm{H} \alpha$ and $\mathrm{H}$ i signals with respect to the brightest levels seen at the (intrinsic) resolution of the simulation by a significant factor. Only the brightest spots in $\mathrm{H} \alpha$ would observable with a Fabry-Pérot interferometer for reasonable integration times, leading to a significant fraction of the ionized gas mass falling below the detection threshold.

\section{RESULTS}

\subsection{Gas Emission}

We follow the evolution of the gas emission over a period of 320 Myr in all 20 models. To illustrate these results, we adopt the model $\tau=1$ as our standard model. The top panels of Figure 5 show the evolution of the $\mathrm{H} \alpha / \mathrm{H}$ i emission at four representative distances $r_{\mathrm{MS}}=55,75,100$, and $150 \mathrm{kpc}$. The

\footnotetext{
9 Note that the LSF of the WHAM spectrometer is only poorly approximated by a Gaussian (Tufte 1997). However, given the typical $\mathrm{H} \alpha / \mathrm{H}$ i $21 \mathrm{~cm}$ line widths $\left(20-40 \mathrm{~km} \mathrm{~s}^{-1}\right)$, this approximation hardly affects our results.
} 

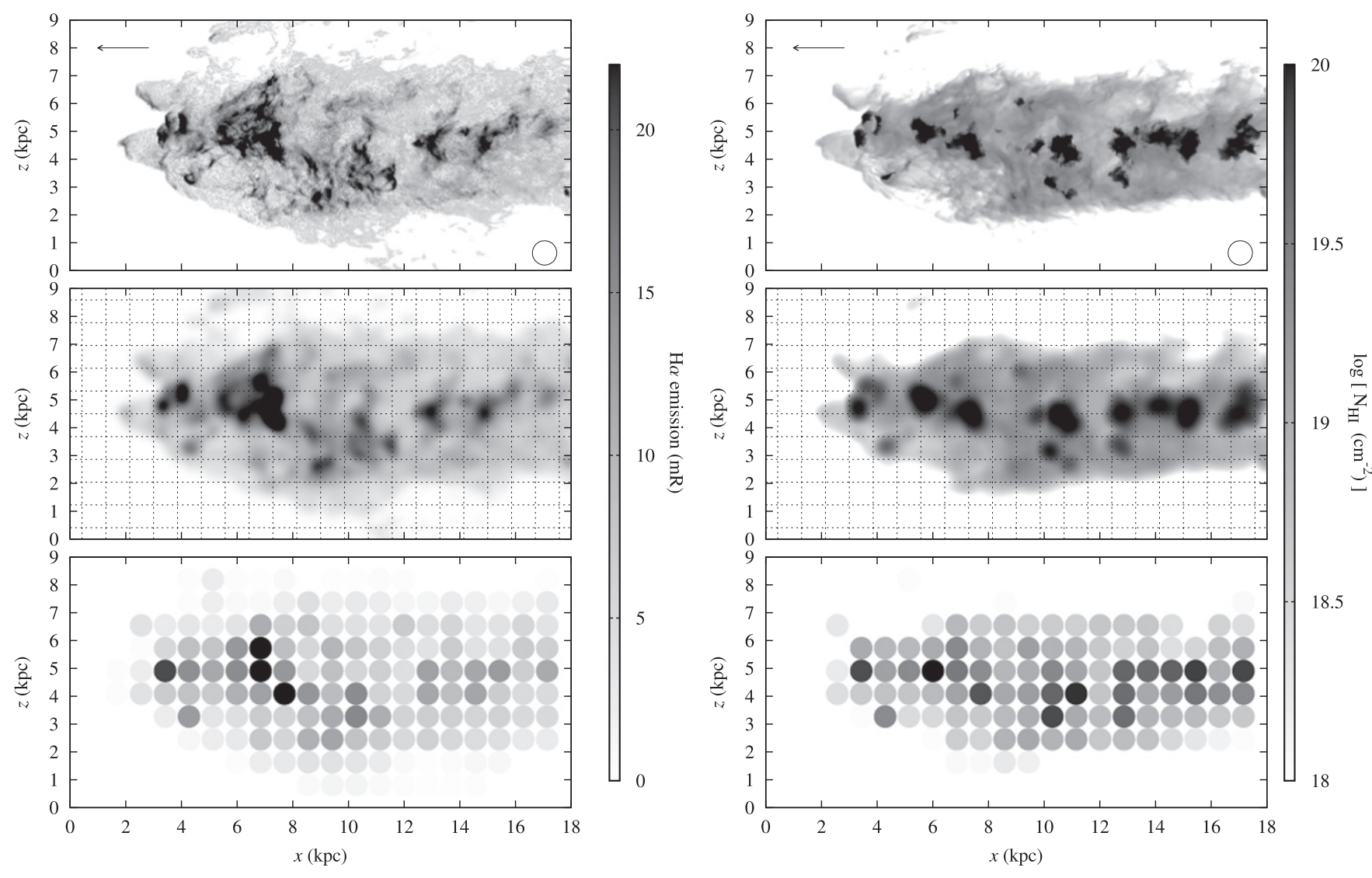

Figure 4. Virtual observation of the $\mathrm{H} \alpha$ (left) and $\mathrm{H}_{\mathrm{I}} 21 \mathrm{~cm}$ (right) emission along the Stream in model $\tau=1$ and $r_{\mathrm{MS}}=55 \mathrm{kpc}$ at $t_{\mathrm{sim}}=170 \mathrm{Myr}$, projected along the $y$ axis. Top: emission at the intrinsic (high) resolution of the simulation. The arrow on the top-left corner indicates the projected Stream's velocity. Middle: the result of spatially smoothing the emission map using a circular Gaussian kernel of size $0.5 / 1^{\circ}$ (FHWM/full width), as indicated by the circle on the bottom-right corner of the top panel. Bottom: emission at different pointings obtained using a beam with an angular diameter of $1^{\circ}$ on the sky. The position of each individual pointing is defined by each cell of the grid overplotted on the middle panel. The grayscale bar to the right of each set of panels indicates the H $\alpha$ intensity in milli-Rayleigh (left) and the $\mathrm{H}$ I column density in $\mathrm{cm}^{-2}$ (right). The full evolution of the $\mathrm{H} \alpha$ surface brightness and the $\mathrm{H} \mathrm{I} \mathrm{column} \mathrm{density} \mathrm{are} \mathrm{available} \mathrm{at} \mathrm{http://www.physics.usyd.edu.au/}$ - tepper/proj_ms.html.

middle panels show the corresponding result for all models $\tau=0.5,0.75,1.0$, and $\tau=1.5$, at a fixed distance $r_{\mathrm{MS}}=55 \mathrm{kpc}$. Note that the comparison of the results for a fixed $\tau$ at different distances allows to assess the effect of the density on the $\mathrm{H} \alpha / \mathrm{H}$ I emission for a fixed temperature, while the comparison of models with different $\tau$ at a fixed distance (and thus density) helps us explore the effect of the temperature.

We find that the gas ionizes quickly ( $\sim 50 \mathrm{Myr})$, and after $\sim 300 \mathrm{Myr}$, the $\mathrm{H}$ I column density has decreased uniformly with time, dropping by nearly an order of magnitude. This can be understood as a consequence of the increasing ionization of the gas due to the interaction with the hot halo gas. The detected column density of the gas farther out is also lower with respect to the intrinsic gas density, which is an effect of the beam dilution. Note that a beam of $1^{\circ}$ diameter samples a region of roughly twice (three times) the size at $r_{\mathrm{MS}}=100 \mathrm{kpc}$ $(150 \mathrm{kpc})$ with respect to $r_{\mathrm{Ms}}=55 \mathrm{kpc}$.

Similarly, the highest $\mathrm{H} \alpha$ emission-this is, the maximum value at each given time-comes from the gas which is closest. In contrast to the behavior of the Hidensity, in this case the effect is governed by the change of halo gas density with distance, and the corresponding change in the strength of the hydrodynamic interaction leading to the shock cascade. What is surprising is that even at the lowest Galactocentric distance of $r_{\mathrm{MS}}=55 \mathrm{kpc}$, the emission never exceeds $\sim 40 \mathrm{mR}$; at $75 \mathrm{kpc}$ it barely reaches $10 \mathrm{mR}$. At even larger distances, $r_{\mathrm{MS}} \gtrsim 100 \mathrm{kpc}$, the emission is dominated by the recombination of the gas ionized by the cosmic UVB. Note that often we do find in our simulation pixels with $\mu_{\mathrm{H} \alpha}>30 \mathrm{mR}$, and occasionally on the order of $\sim 100 \mathrm{mR}$, but their strong signal is washed out as a result of the beam smearing (Figure 4; see also Section 4.4). The similarity in $\mathrm{H} \alpha$ emission across models with different $\tau$ shows that these results are insensitive to variations in the halo gas temperature by factors of a few. This indicates that the $\mathrm{H} \alpha$ emission is dominated by the gas ionized through cloudcloud collisions that trigger the shock cascade, rather than the gas ionized by the interaction with the hot halo. The insignificance of the halo gas temperature together with the fact that the virial temperature across the DM halo models presented previously is very similar (Table 1) makes the choice of the DM halo model irrelevant, as long as the corresponding gas density profiles are similar.

We see that the maximum level of $\mathrm{H} \alpha$ emission at any reasonable distance is comparable to, or even less than, the emission induced by the Galactic ionizing starlight $\sim 20-40$ $\mathrm{mR}[d / 55 \mathrm{kpc}]^{-2}$. This is the reason for us to ignore this component in our models, since it would otherwise outshine the emission produced by the shock cascade. Taking the contribution of the Galactic UV into account would elevate the emission at $55 \mathrm{kpc}$ to $\sim 70 \mathrm{mR}$, and to $\sim 30 \mathrm{mR}$ at $75 \mathrm{kpc}$, which are significantly lower than the Stream's emission observed over the SGP and at the tail of the Stream at 

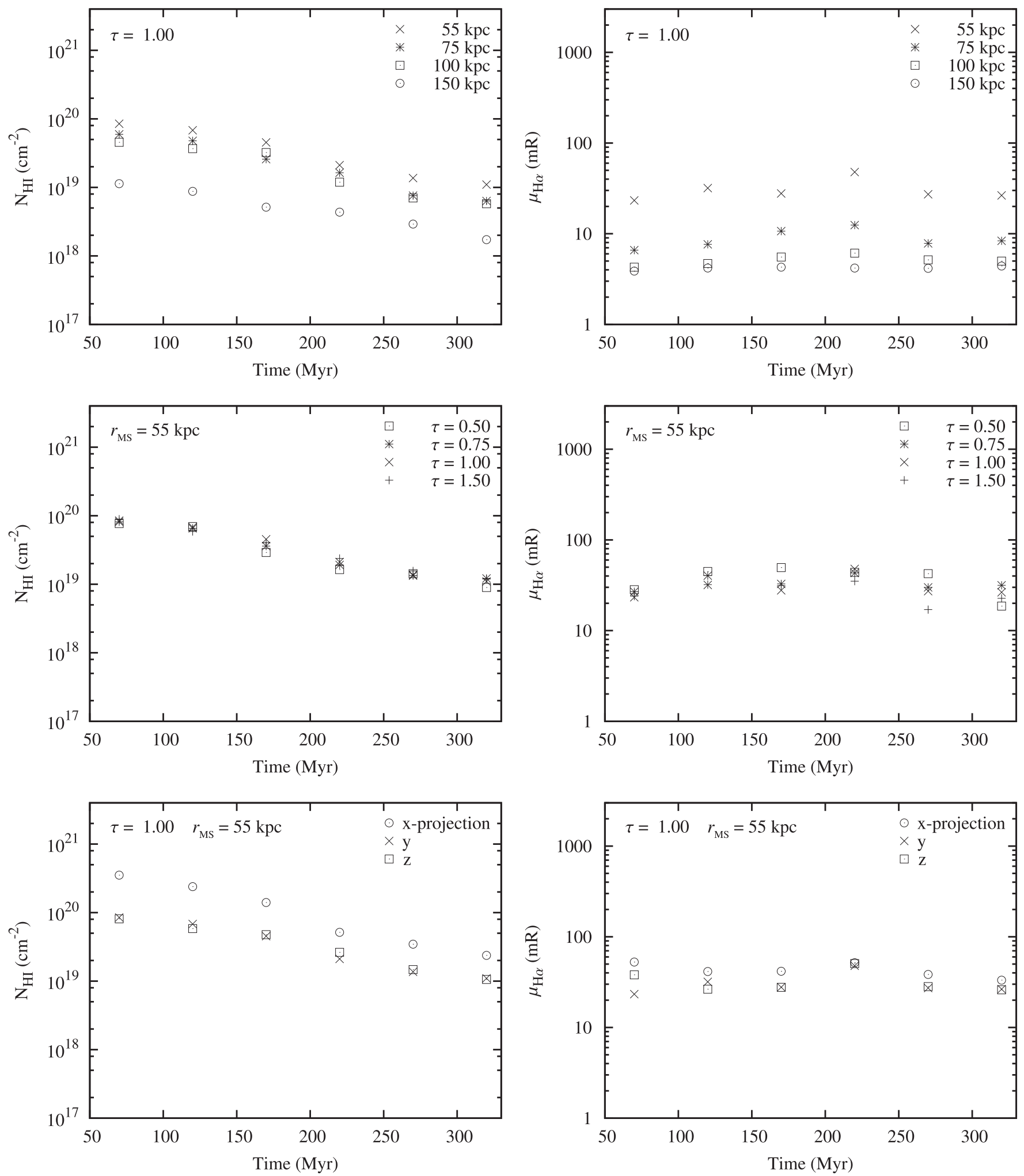

Figure 5. Evolution of the $\mathrm{H}$ i column density (left) and the $\mathrm{H} \alpha$ surface brightness (right). The top panels correspond to the results for model $\tau=1$, and a range in Galactocentric distance $r_{\mathrm{MS}}$ (indicated by the legend). The middle panels show the result at a fixed Galactocentric distance $r_{\mathrm{MS}}=55 \mathrm{kpc}$, for different $\tau$ (indicated by the legend). Each data point in the left (right) panels corresponds to the 90 (100) percentile of the distribution of intensities at that particular time and distance. The four top panels correspond to the results obtained from projecting the simulation cube along the $y$-axis. The two bottom panels illustrate the difference between projections along all three orthogonal axes for model $\tau=1$ at $55 \mathrm{kpc}$.

$l_{\mathrm{M}} \approx 260^{\circ}$, respectively (Bland-Hawthorn et al. 2013). It is, however, unclear at this point how the ionizing effect of the Galactic UV included self-consistently at runtime would affect these limits.
Given that observations performed with the WHAM instrument typically reach a sensitivity of $\gtrsim 30 \mathrm{mR}$, the Stream gas in our models would be essentially undetectable (ignoring for the moment the contribution that results from the Galactic 

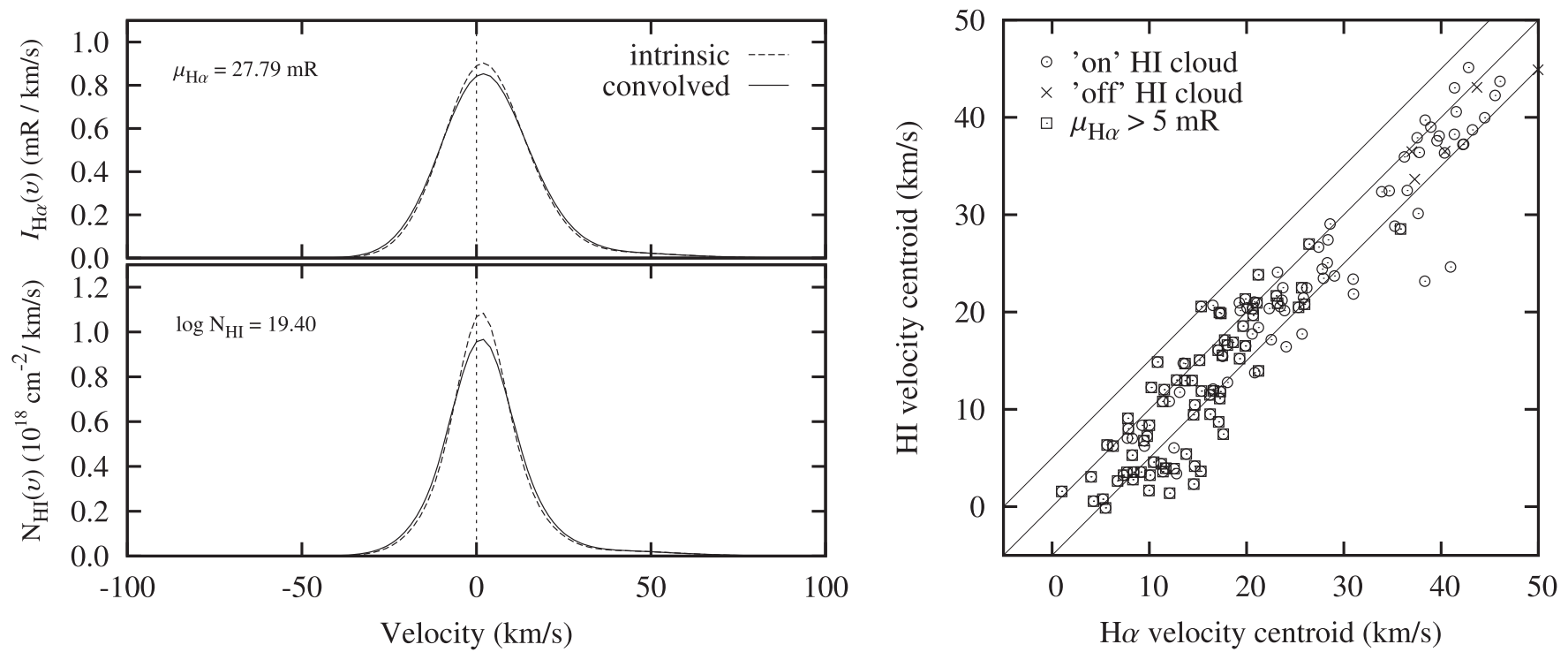

Figure 6. Gas kinematics in model $\tau=1$ and $r_{\mathrm{Ms}}=55 \mathrm{kpc}$ at $t_{\mathrm{sim}}=170 \mathrm{Myr}$. Left: emission spectra observed at the pointing with the brightest H $\alpha$ emission shown in the bottom left panel of Figure 4. We show for comparison the intrinsic line profile and the line profile convolved with a Gaussian LSF with a FWHM $=12 \mathrm{~km} \mathrm{~s}{ }^{-1}$. Right: line of sight velocity centroid of the $\mathrm{H} \alpha$ intensity detected "on" and "off" $\mathrm{H}$ I clouds (see the text for details). The solid diagonal lines indicate the identity line and $\mathrm{a} \pm 5 \mathrm{~km} \mathrm{~s}^{-1}$ range around this line.

ionizing field). In contrast, and considering that the sensitivity of e.g the GASS survey is roughly $N_{\mathrm{H}}=1.6 \times 10^{18} \mathrm{~cm}^{-2}$, the gas at distances $d \lesssim 100 \mathrm{kpc}$ would be bright in $\mathrm{H} \mathrm{I} 21 \mathrm{~cm}$, and marginally detectable at $d \sim 150 \mathrm{kpc}$, even after $300 \mathrm{Myr}$.

We find that much of the gas dislodged from the main body of our model Stream is low density material that mixes rapidly with the halo gas, thereby being heated (and thus ionized) to temperatures well above $10^{5} \mathrm{~K}$, which are on the order of the temperature expected for turbulent mixing (Begelman \& Fabian 1990). At these temperatures, the $\mathrm{H} \alpha$ emissivity drops by nearly two orders of magnitude with respect to its value $10^{4} \mathrm{~K}$ (see Equation (17)), and the ionized gas becomes thus practically invisible in $\mathrm{H} \alpha$. Only the gas ionized by cloudcloud collisions remains at relatively low temperatures $\left(\sim 10^{4} \mathrm{~K}\right)$, and recombines quickly, thus providing the strongest $\mathrm{H} \alpha$ signal. However, the fraction of warm ionized gas is very low overall, and thus is the corresponding $\mathrm{H} \alpha$ signal. Hence, only a mechanism such as slow shocks which is able to ionize a significant fraction of the gas without increasing its temperature far above $10^{4} \mathrm{~K}$ will lead to significant $\mathrm{H} \alpha$ emission.

As the reader may recall, all the above results correspond to virtual observations where the simulation cube has been projected along the $y$-axis. Given that this choice is somewhat arbitrary, we calculate the corresponding results for projections along all three orthogonal axes for model $\tau=1$ at $55 \mathrm{kpc}$. The outcome of this exercise is summarized in the bottom panels of Figure 5. We have checked that the results are essentially the same for all other models. The Hicolumn density is consistently highest when observed along the $x$-axis, given that this axis coincides with the axis of symmetry of the initially cylindrical gas configuration in our setup, and the sightline traverses a larger path across the $\mathrm{H}$ I cloud. The projections along the $y$ and $z$ axes yield nearly identical results, as expected from the symmetry of the initial cloud structure.

In our experiment, the projection along the $x$-axis is equivalent to observing the Stream "face-on." Hence, one would naïvely expect that the $\mathrm{H} \alpha$ emission should be highest when projecting along this direction. But surprisingly, the $\mathrm{H} \alpha$ intensity in our models is very similar regardless of the projection, being only slightly stronger when viewing the gas cloud face-on. This implies that the choice of projection axis to compute virtual observations is essentially irrelevant. Moreover, since different projections effectively imply significant variations in the impact angle, our choice of a particular value for $\vartheta$ turns to be irrelevant as well, as far as the $\mathrm{H} \alpha$ intensities are concerned.

\subsection{Gas Kinematics}

The kinematics of the warm neutral and ionized gas phases as traced by $\mathrm{H} \alpha$ and $\mathrm{HI}$ emission provide insight into the mechanism ionizing the Stream. We explore this using the spectra of our model identified by $\tau=1$ and $r_{\mathrm{MS}}=55 \mathrm{kpc}$ at $t_{\text {sim }}=170 \mathrm{Myr}$. Note that the results, with the exception of the $\mathrm{H} \alpha$ emission strength, are virtually identical for all other models. Examples of a strong $\mathrm{H} \alpha$ and $\mathrm{H}_{\mathrm{I}} 21 \mathrm{~cm}$ emission lines is shown in the top panel of Figure 6. These correspond to the pointing with the brightest $\mathrm{H} \alpha$ emission shown in the bottom panel of Figure 4. The integrated strength of the line is indicated in each case in the top-left corner. For reference, the typical sensitivity of WHAM $(30 \mathrm{mR})$ translates into a spectral sensitivity of $0.5 \mathrm{mR} / \mathrm{km} \mathrm{s}^{-1}$, assuming a typical line width of $30 \mathrm{~km} \mathrm{~s}^{-1}$. Similarly, the sensitivity of the GASS survey $\left(N_{\mathrm{H}}=1.6 \times 10^{18} \mathrm{~cm}^{-2}\right)$ corresponds to a spectral sensitivity of $10^{17} \mathrm{~cm}^{-2} / \mathrm{km} \mathrm{s}^{-1}$. Thus, while the $\mathrm{H} \alpha$ emission in this case is marginally above the WHAM detection threshold, the corresponding $\mathrm{H}_{\mathrm{I}} 21 \mathrm{~cm}$ signal would be comfortably detected in a survey similar to GASS. We find typical line widths of 20 $-30 \mathrm{~km} \mathrm{~s}^{-1}$ (FWHM), which are consistent with observations (Putman et al. 2003).

We study the difference in the kinematics of the warm neutral and ionized gas by comparing the sightline velocity centroid of the $\mathrm{H} \alpha$ emission to the $\mathrm{HI}_{\mathrm{I}} 21 \mathrm{~cm}$ emission, distinguishing between pointings "on" and "off" the $\mathrm{H}_{\mathrm{I}}$ clouds. In this context, "on" ("off") means that the H I $21 \mathrm{~cm}$ 


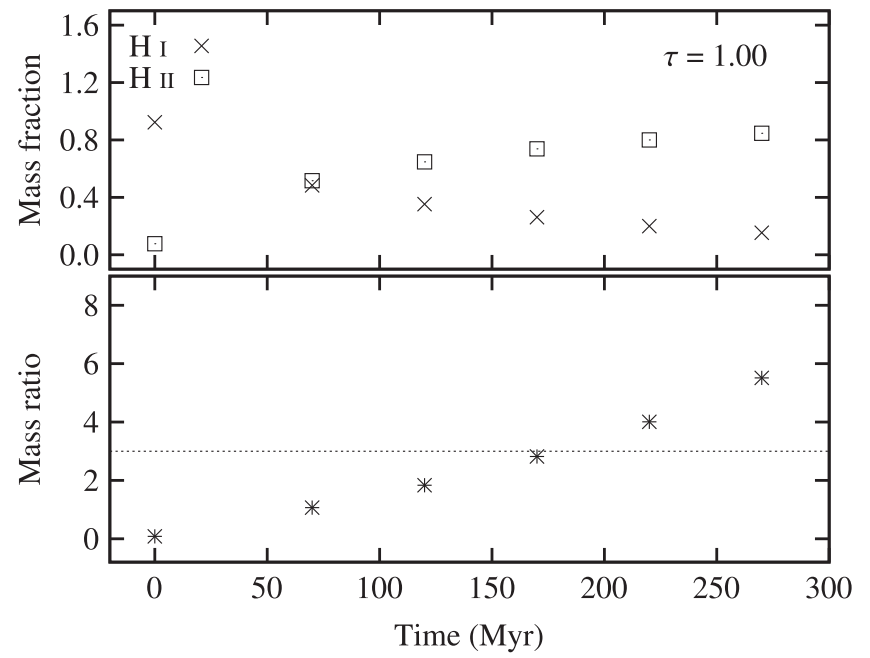

Figure 7. Evolution of the $\mathrm{H}_{\mathrm{I}}$ and $\mathrm{H}$ II mass fractions in the $\tau=1$ model Stream at $r_{\mathrm{MS}}=55 \mathrm{kpc}$, projected along the $y$-axis. The top panel shows the evolution of the individual mass fractions $M_{X} / M_{\text {tot }}$, where $X \in\{\mathrm{H} \mathrm{I}, \mathrm{H}$ II $\}$ and $M_{\mathrm{tot}}=M_{\mathrm{HI}}+M_{\mathrm{HI}}$. The bottom panel shows the evolution of the $\mathrm{H}$ II to $\mathrm{H}$ I mass ratio. The dashed horizontal line indicates the ratio $\mathrm{H}$ II:H I $=3$ inferred from observations (Fox et al. 2014). The results are essentially the same for all other models, and are therefore not shown.

emission is above (below) the GASS detection limit $N_{\mathrm{H} \text { I }}=1.6 \times 10^{18} \mathrm{~cm}^{-2}$. Note that the line centroid corresponds to the intensity-weighted mean velocity. In addition, we flag those pointings where the $\mathrm{H} \alpha$ emission is above the level expected from ionization by the UBV (Figure 6, bottom panel). We do not find a significant difference between the sightline velocities of the warm neutral and ionized gas phases; their respective velocity centroids agree within $\pm 5 \mathrm{~km} \mathrm{~s}^{-1}$, as observed (Putman et al. 2003; Barger et al. 2015). There is a tendency for the $\mathrm{H} \alpha$ lines to have slightly higher velocity centroids. This arises from the higher line asymmetry resulting from a more extended emission along the sightline. It is interesting that we barely find any $\mathrm{H} \alpha$ emission above $5 \mathrm{mR}$ detached from $\mathrm{H}_{\mathrm{I}} 21 \mathrm{~cm}$ emission. Also, the velocity of this strong $\mathrm{H} \alpha$ emission is generally low $\left(v \lesssim 20 \mathrm{~km} \mathrm{~s}^{-1}\right)$, with a tendency for the strongest emission to have the lowest velocities (not shown), indicating that the strong $\mathrm{H} \alpha$ emission is physically associated with the $\mathrm{H}$ Igas. This coincidence in both velocity and physical space of the $\mathrm{H} \alpha / \mathrm{H}$ i signal is-recall the weak dependence of the $\mathrm{H} \alpha$ emission on the halo temperature-another characteristic signature of the shock cascade.

\subsection{Gas Ionization Timescales}

It has recently been inferred that the mass of the ionized gas kinematically associated to the MS is roughly three times larger than its H i mass (Fox et al. 2014). Here, we briefly explore the evolution of the ionized and warm-neutral gas mass fractions, using the $\mathrm{H}$ II and $\mathrm{H}$ i masses in as a proxy for the ionized and warm-neutral gas phases, respectively. Again, we focus on the results obtained from our models characterized by $\tau=0.50$, $0.75,1.00$, and $r_{\mathrm{MS}}=55 \mathrm{kpc}$. Note that the results are qualitatively the same for all other models. Figure 7 shows the evolution of the individual mass fractions $f_{X} \equiv M_{X} / M_{\text {tot }}$, where $X \in\left\{\mathrm{H}_{\mathrm{I}}, \mathrm{H}_{\mathrm{II}}\right\}$ and $M_{\mathrm{tot}}=M_{\mathrm{H}_{\mathrm{I}}}+M_{\mathrm{H} \text { II }}$, as well as the evolution of the $\mathrm{H}$ II to $\mathrm{H}$ Imass ratio. We include for reference the inferred value of the ratio $\mathrm{H}$ п: $\mathrm{H}_{\mathrm{I}}=3$. We find that the gas evolves on a typical timescale of $100 \mathrm{Myr}$, which is consistent with the time estimate that results from assuming that the cloud is disrupted by the action of Kelvin-Helmholtz instabilities and our adopted (initial) value of $\eta$. After this period, the ionization effect of the halo-cloud and cloud-cloud interaction leads to a reduction of the warm neutral gas mass by half. The $\mathrm{H}$ II: $\mathrm{H}$ I mass ratio increases rapidly with time, and the inferred $\mathrm{H}$ II: $\mathrm{H}$ I mass ratio of 3 is reached after $\sim 170 \mathrm{Myr}$.

Since our simulations assume free boundary conditions, it is difficult to quantify with precision how much of the neutral gas is ionized and how much simply escapes the simulation volume. Nonetheless, we estimate that only a negligible fraction of the warm-neutral gas is lost by $t_{\text {sim }} \lesssim 270 \mathrm{Myr}$. Therefore, the ionization of the gas due to interactions with the hot halo gas, and due to cloud-cloud interactions leads to a strong evolution of the mass fractions in the neutral and ionized phases. The relatively short survival timescale implies the requirement for a continuous replenishment of gas from the MC to the Stream (Bland-Hawthorn et al. 2007).

Note that for ease of discussion, in the following section we shall refer to the set of models discussed in the last sections, defined by $n(55 \mathrm{kpc})=2 \times 10^{-4} \mathrm{~cm}^{-3}$, as the "standard" model set.

\subsection{Conservative Departures from the Standard Models}

Given the results of the last sections, our standard set of models appears to indicate that the shock cascade fails to produce the mean level of $\mathrm{H} \alpha$ emission $(\sim 160 \mathrm{mR}$; BlandHawthorn et al. 2013) observed along the MS. But there are two factors that deserve closer consideration. First, a halo density at any given distance within $r_{\text {vir }}$ higher than implied by our standard models could enhance the onset and development of hydrodynamic instabilities (Kelvin-Helmholtz), thus promoting the shock cascade and the resulting $\mathrm{H} \alpha$ emission. Second, an increase in the beam resolution would certainly diminish the smearing effect on bright spots that have characteristic sizes significantly smaller than the beam. Consider, for instance, that a beam with a diameter of $10^{\prime}$ samples a region that is nearly 10 times smaller than a $1^{\circ}$ beam at $r_{\mathrm{MS}}=55 \mathrm{kpc}$. Indeed, the brightest $\mathrm{H} \alpha$ observations along the Stream have been obtained with spectroscopy over smaller apertures $\left(3^{\prime}-10^{\prime}\right)$ than the WHAM survey (e.g., Putman et al. 2003).

Therefore, it is important to extend the parameter space of our study, in terms of both the halo density and the adopted beam size. We now increase the normalization of the halo gas density at $55 \mathrm{kpc}$ by a factor of 2 , i.e., $n$ $(55 \mathrm{kpc})=4 \times 10^{-4} \mathrm{~cm}^{-3}$, but with initial conditions and set up which are otherwise identical in every aspect to the standard models. These shall be referred to as the "extended" models. In addition to increasing the density, we produce a new set of virtual observations for the standard models, adopting a smaller beam size (i.e., a higher resolution) with a diameter of $\theta=10^{\prime}$ (rather than $1^{\circ}$ ), and two sets of virtual observations for the extended models, adopting either a low $\left(1^{\circ}\right)$ or a high $\left(10^{\prime}\right)$ resolution beam. We shall refer to these as the "low-resolution" and "high-resolution" models, respectively, keeping in mind that is not the actual hydrodynamical model, but the virtual observation, to which the resolution refers. 

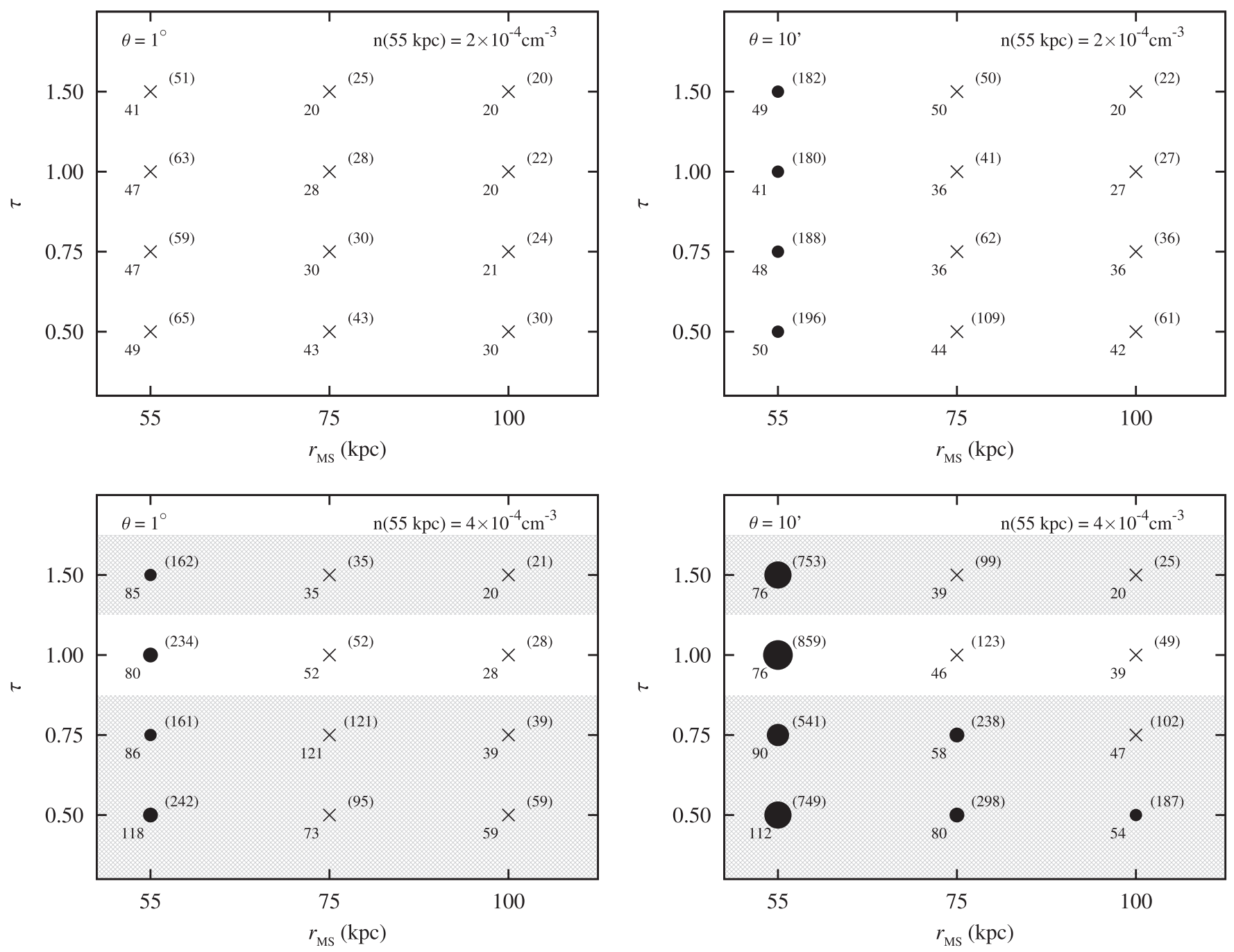

Figure 8. Overview of model results. In any panel, a model is identified by the set of parameters $\left\{n(55 \mathrm{kpc}), \theta, \tau, r_{\mathrm{MS}}\right\}$. The corresponding data point consists of a symbol $(\bullet$ or $\times$ ), and two numerical values. The value to the top-right of a given symbol (in parentheses) indicates the maximum H $\alpha$ emission (mR) observed in the full time lapse of $320 \mathrm{Myr}$ in that particular model, while the value to the bottom-left indicates the $\mathrm{H} \alpha$ emission $(\mathrm{mR})$ at the 90 percentile level. A circle $(\bullet)$ indicates whether the maximum $\mathrm{H} \alpha$ intensity exceeds $160 \mathrm{mR}$; a cross $(\times)$ signals failure to do so. The circle diameter is roughly proportional to the maximum H $\alpha$ intensity in each case. The gray hatched area highlights models that are inconsistent with the halo mass constraint (see the text for details). Note that the top (bottom) panels correspond to models where $n(55 \mathrm{kpc})=2 \times 10^{-3} \mathrm{~cm}^{-3}\left(4 \times 10^{-3} \mathrm{~cm}^{-3}\right)$, and left (right) panels correspond to models where a low (high) resolution beam with diameter $\theta=1^{\circ}\left(10^{\prime}\right)$ has been adopted to produce virtual observations. All models at $r_{\mathrm{MS}}>100 \mathrm{kpc}$ have been omitted since their respective H $\alpha$ emission is weak.

Note that the density profiles implied by the extended models are fairly consistent with observations (Figure 3, bottom-left panel), although there is no model which agrees with the data over the full range in distance. However, the enclosed gas mass in the halo within $r_{\text {vir }}$ resulting from each of these density profiles is larger than the the mean range of masses inferred from observations, and-with the exception of the $\tau=1$ extended model-they are all inconsistent with the mass limit imposed by the universal baryon-to-total-mass fraction (Figure 3, bottom-left panel). Hence, all the extended models but the $\tau=1$ model, may be deemed "unphysical." Nonetheless, it is still of interest to explore the $\mathrm{H} \alpha$ intensity in these types of models, as will be discussed later.

Each of the models in either the standard or the extended set is run for a total simulation time of $t_{\text {sim }}=320 \mathrm{Myr}$, and a virtual observation of the $\mathrm{H} \alpha$ intensity of the gas at the appropriate resolution is produced every $\Delta t_{\text {sim }}=10 \mathrm{Myr}$. Therefore, for each model and at each time step we obtain a whole new distribution of $\mathrm{H} \alpha$ intensities. In order to deal with the overwhelming amount of information, and to make a meaningful comparison between models, we opt for the following approach: since the total time lapse $t_{\text {sim }}$, and the choice of output time step are somewhat arbitrary, for each model we single out the snapshot at which the maximum $\mathrm{H} \alpha$ intensity anywhere in the gas (i.e., at any beam pointing) is largest. This is further justified by the fact that the maximum $\mathrm{H} \alpha$ emission does not evolve strongly with time (Figure 5). Note that the snapshot thus selected will in general be different for each model. For this particular snapshot and model, we also obtain the value of the emission at the 90 percentile level of the corresponding distribution. We then assess the performance of each model simply by comparing both the maximum $\mathrm{H} \alpha$ emission to the mean level of $\mathrm{H} \alpha$ emission $(\sim 160 \mathrm{mR})$ observed along the MS. The result of these approaches applied to both the standard and the extended models is collected in Figure 8.

There, the top panels correspond to the standard models, and the bottom panels show the results for the extended set; the left 
(right) panels correspond to the low (high) resolution cases. In any panel, each "data point" corresponds to a particular model identified by $\left\{n(55 \mathrm{kpc}), \theta, \tau, r_{\mathrm{MS}}\right\}$, and it consists of a symbol (circle or cross), and two numerical values. The value to the top-right of a given symbol (in parentheses) indicates the maximum $\mathrm{H} \alpha$ emission (in $\mathrm{mR}$ ), while the value to the bottomleft indicates the value at the 90 percentile level. A circle indicates whether the maximum $\mathrm{H} \alpha$ intensity exceeds $160 \mathrm{mR}$; a cross signals failure to do so. The circle diameter is roughly proportional to the maximum $\mathrm{H} \alpha$ intensity in each case. In addition, we have grayed hatched the parameter space corresponding to models that are deemed "unphysical" as per the above discussion. As a guide, note that the series of numbers in parentheses, i.e., the maximum $\mathrm{H} \alpha$ intensity, shown on the top-left panel for model $\tau=1$ correspond to the results shown in the top-right panel of Figure 5 at 220 Myr (ignoring the $150 \mathrm{kpc}$ series). Similarly, the maximum $\mathrm{H} \alpha$ intensities at $r_{\mathrm{Ms}}=55 \mathrm{kpc}$ for all models correspond to the results shown in the bottom-right panel of Figure 5 at $170 \mathrm{Myr}$ for $\tau=0.5$, and at 220 Myr for all the other models.

Apparently, both a higher density and an improved resolution enhance the $\mathrm{H} \alpha$ emission, but in different ways. On the one hand, increasing the density shifts the overall $\mathrm{H} \alpha$ intensity toward the high-end. This can be seen by comparing the maximum $\mathrm{H} \alpha$ intensity (and the 90 percentile) between the standard and the extended models, which are roughly a factor of 2-3 higher in the latter. Increasing the resolution, on the other hand, boosts only the maximum $\mathrm{H} \alpha$ emission, without significantly affecting the distribution of intensities as a whole, as can be judged by comparing the values at the 90 percentile level.

The standard models at low resolution (top-left panel) fail dramatically at any distance in matching the Stream's mean emission. Both the standard model at high resolution (top-right) and the extended model at low resolution (bottom-left) result in $\mathrm{H} \alpha$ intensities in the $100 \mathrm{mR}$ regime only in the near field at $55 \mathrm{kpc}$. In this sense, both a higher halo density model, and virtual observations with a high resolution are equally crucial factors in pushing the $\mathrm{H} \alpha$ emission toward higher levels. It is worth mentioning at this point that the original model by Bland-Hawthorn et al. (2007) — an instance of a "standard" model in our terminology-implicitly assumed an infinite resolution, and was therefore capable of reproducing $\mathrm{H} \alpha$ emission at levels of a few hundred milli-Rayleigh at $55 \mathrm{kpc}$.

The extended, high resolution models (bottom-right) are the most promising of all the models considered here. In the near field, the shock cascade in all these models results in $\mathrm{H} \alpha$ intensities which reach, or even exceed, the highest levels of $\sim 700 \mathrm{mR}$ observed along the MS over the SGP. In the far field $\left(r_{\mathrm{MS}} \sim 75-100 \mathrm{kpc}\right)$, two of the models $(\tau=0.5$ and $0.75)$ produce $\mathrm{H} \alpha$ emission consistent with the mean emission of $\sim 160 \mathrm{mR}$ observed along the Stream. However, the success of these models comes at a cost. The increase in the halo density is accompanied by an increase in the halo mass. This makes all but the $\tau=1$ model be inconsistent with the limit on the gas mass of the Galaxy imposed by the cosmic fraction of baryons relative to the total mass. Given that the isothermal sphere model yields an upper limit on the halo gas density at any distance and for a given gas mass, the situation is even more unfortunate for any other reasonable DM halo model.

\section{DISCUSSION}

Within the context of the "shock cascade" model, we have shown that the interpretation of the MS optical emission $(\approx 100$ $-200 \mathrm{mR}$ ) away from the MCs may still work for the updated parameters of the Galaxy under a narrow set of conditions. Conventionally, the MS was assumed to be on a circular orbit at the midpoint of the LMC and SMC ( $d \approx 55 \mathrm{kpc})$. At this distance, for a smooth halo density profile, as long as the coronal halo density satisfies $2 \times 10^{-4}<\left(\mathrm{n} / \mathrm{cm}^{-3}\right)<$ $4 \times 10^{-4}$, the shock cascade generates sufficient $\mathrm{H} \alpha$ emission to explain the observations. The upper limit on density ensures that the mass of the corona (when including the other baryonic components) does not exceed the baryonic mass budget of the Galaxy.

In recent years, the first accurate measurements of the MC's proper motions, combined with a smaller estimate of the Galaxy's total mass, have led to a major revision of their binary orbit about the Galaxy. A highly elliptic orbit is now favored by most researchers, which pushes the Stream's mean distance further out than the conventional assumption. For a smooth halo, the lower density limit of the above range can occur at $d \approx 75 \mathrm{kpc}$ without violating the constraint on the baryonic mass budget. If the Stream's mean distance (especially over the SGP) happens to exceed this limit, then we are forced to either reject the model, or consider more complex density distributions for the Galactic corona (see below).

An alternative interpretation of the Stream's optical emission has recently been put forward in the context of the Galaxy's nuclear activity. The energetic bubbles observed with the Fermi-LAT in gamma rays ( $\mathrm{Su}$ et al. 2010) indicate that a powerful event has taken place at the nucleus in the recent past. If the gamma rays are produced through inverse Compton upscattering of soft photons, this event can be dated to 1-3 Myr ago (Guo \& Mathews 2012). Within the context of this model, Bland-Hawthorn et al. (2013) show that the Stream $\mathrm{H} \alpha$ emission can also be explained by accretion-disk driven ionization for Stream distances of $100 \mathrm{kpc}$ or more over the poles. The recent discovery of high ionization species (e.g., Si IV, C IV) over the SGP (Fox et al. 2015) may lend further support to this model. On the other hand, Bland-Hawthorn et al. (2007) provide diagnostics of slow shocks (e.g., Balmer decrement) that are likely to be observable along the MS in future observing campaigns. If enhanced Balmer decrements $(\mathrm{H} \alpha / \mathrm{H} \beta \gtrsim 3)$ are confirmed along the Stream, then some variant of the shock cascade model may be needed.

There is now increasing evidence that the CGM of low redshift galaxies is multi-phase, with a comparable fraction of baryons both in a hot and a warm phase (Werk et al. 2014). Modern simulations of the CGM also suggest that the hot halos of galaxies are likely to be heavily structured, at least during a major phase of gas accretion. While CDM accretion may be isotropic on average, individual events involving massive systems are not, as clearly demonstrated by the MCs in orbit around the Galaxy, or the Sgr dwarf which extends through much of the halo (Ibata et al. 1994). The mass of this system was probably comparable to the LMC and may well have retained gas before being tidally disrupted. A more recent accretion event is attested by the massive H i stream, the Smith Cloud $\left(\gtrsim 2 \times 10^{6} M_{\odot}\right)$, that is presently being stripped and ablated by the corona (Bland-Hawthorn et al. 1998; Lockman et al. 2008; Nichols et al. 2014). 
We may need therefore to consider the possibility that the corona is inhomogeneous rather than smooth. This would allow for significant density variations along different directions and at different distances, without violating constraints on the total baryonic mass of the Galaxy. In turn, based on our extended models, this would restore the shock cascade as a viable model to explain the $\mathrm{H} \alpha$ emission, allowing the Stream to lie at $\sim 100 \mathrm{kpc}$ the SGP as predicted by dynamical models (e.g., Guglielmo et al. 2014). In this scenario, we may envisage the strong optical emission along the Stream as a result of the Stream's gas colliding with high-density debris of past accretion events along its orbit. At this point, however, it is not clear what a suitable model for an inhomogeneous hot halo might be. We will address this in future work.

If confirmed, the larger distance to the Stream of $100 \mathrm{kpc}$ would lift its mass to roughly $8 \times 10^{9} M_{\odot}$, comparable to the total coronal gas mass. If it all breaks down, it would roughly double the mass of the corona, at least for a while. We thus speculate that the halo of the Galaxy is substantially massloaded with gas lost by smaller accreted systems. The interaction with the hot halo may prevent this gas from cooling sufficiently to condense and "rain" down on the disk. Such a process is analogous to the meteorologic phenomenon know as virga, a type of atmospheric precipitation that evaporates while dropping and thus fails to reach the ground. The heavy halo may thus serve as a huge reservoir, from which gas may eventually be forced out by the strong interaction at the diskhalo interface (e.g., Marinacci et al. 2010).

T.T.G. acknowledges financial support from the Australian Research Council (ARC) through a Super Science Fellowship and an Australian Laureate Fellowship awarded to J.B.H.

\section{APPENDIX A \\ $\mathrm{H} \alpha$ RECOMBINATION COEFFICIENT}

We describe the temperature dependence of the hydrogen total case $\mathrm{B}$ recombination coefficient, $\alpha_{B}$, and of the effective $\mathrm{H} \alpha$ recombination coefficient, $\alpha_{B}^{(\mathrm{H} \alpha)}$, with the generic fitting formula (Pequignot et al. 1991):

$$
\alpha(T)=\alpha_{0} \times \frac{(1+c)\left(T / 10^{4} K\right)^{b}}{1+c\left(T / 10^{4} K\right)^{d}} .
$$

The parameter values appropriate in each case are, respectively, $\alpha_{0}=2.585 \times 10^{-13} \mathrm{~cm}^{3} \mathrm{~s}^{-1}, b=-0.6166, c=0.6703$, and $d=0.5300$, and $\alpha_{0}=1.169 \times 10^{-13} \mathrm{~cm}^{3} \mathrm{~s}^{-1}, b=-0.648$, $c=1.315$, and $d=0.523$. Note that this formula is accurate to $2 \%$ in the range $40 \mathrm{~K}<T<2 \times 10^{4} \mathrm{~K}$.

\section{APPENDIX B

PHOTOIONIZATION-INDUCED H $\alpha$ EMISSION

We include in our model the contribution of the cosmic UVB ionizing radiation, which leads to a low, but non-negligible level of $\mathrm{H} \alpha$ emission along the Stream. For simplicity, we assume that the gas is highly ionized $\left(n_{\mathrm{H}} \approx n_{\mathrm{H}}\right)$; ; and photoionization equilibrium, which implies that the ionization and recombination events balance each other:

$$
\left(n_{e} n_{\mathrm{H} \mathrm{II}}\right) \alpha_{B}=n_{\mathrm{HI}} \Gamma_{\mathrm{HI}} \text {. }
$$

Here, $\Gamma_{\mathrm{H}_{\mathrm{I}}}$ is the $\mathrm{H}_{\mathrm{I}}$ photoionization rate in units of photon per atom per second; $J_{\nu}$ is the angle-averaged specific intensity of the
$\mathrm{UVB} ; \nu_{\mathrm{LL}} \approx 3.29 \times 10^{15} \mathrm{~Hz}$ is the minimum photon frequency required to ionize hydrogen; and $\sigma_{\mathrm{H}}(\nu) \approx \sigma_{0}\left(\nu / \nu_{\mathrm{LL}}\right)^{3}$ is the hydrogen ionization cross-section with $\sigma_{0}=6.3 \times 10^{-18} \mathrm{~cm}^{2}$.

The $\mathrm{H} \alpha$ emission induced by the metagalactic ionizing radiation field along the sightline is thus given by Equation (14), where the fraction of recombinations that produce an $\mathrm{H} \alpha$ photon is

$$
f_{\mathrm{H} \alpha}(T) \equiv \frac{\alpha_{B}^{(\mathrm{H} \alpha)}(T)}{\alpha_{B}(T)} \approx 0.452 g(T),
$$

Here, $g$ is a monotonically decreasing function of temperature (see Equation (17))

$$
g(T)=\left(\frac{1+c}{1+c^{\prime}}\right)\left[\frac{1+c^{\prime}\left(T / 10^{4} K\right)^{d^{\prime}}}{1+c\left(T / 10^{4} K\right)^{d}}\right]\left(T / 10^{4} K\right)^{b-b^{\prime}},
$$

which satisfies $g\left(10^{4} \mathrm{~K}\right) \equiv 1, \quad$ and $\quad g \in(0.6,1.3)$ for $T \in\left[10^{3}, 10^{6}\right] \mathrm{K}$.

In general, $f_{\mathrm{H} \alpha}$ (through $T$ ) and $n_{\mathrm{H}_{\mathrm{I}}}$ both vary along the sightline. However, an estimate of the $\mathrm{H} \alpha$ signal resulting from the ionization by the cosmic UVB of gas at can be obtained assuming the gas temperature to be uniform along the sightline. In this case, and inserting the appropriate numerical values we get

$$
\mu_{\mathrm{H} \alpha}{ }^{(\text {phot })} \approx 452 \mathrm{mR}\left(\frac{N_{\mathrm{H} \mathrm{I}}}{10^{18} \mathrm{~cm}^{-2}}\right)\left(\frac{\Gamma_{\mathrm{H} \mathrm{I}}}{10^{-12} \mathrm{~s}^{-1}}\right),
$$

where $N_{\mathrm{H}_{\mathrm{I}}}$ is the integral of $n_{\mathrm{H} \text { I }}$ along the sightline. Hence, for an $\mathrm{H}_{\mathrm{I}}$ ionization rate $\Gamma_{\mathrm{H}_{\mathrm{I}}} \sim 10^{-13} \mathrm{~s}^{-1}$ at $z=0$ and gas at $10^{4} \mathrm{~K}$ with $N_{\mathrm{H}_{\mathrm{I}}} \sim 10^{17} \mathrm{~cm}^{-2}$, the $\mathrm{H} \alpha$ signal resulting from the ionization by the cosmic UVB of gas at $10^{4} \mathrm{~K}$ is roughly $5 \mathrm{mR}$.

Since we are not performing proper radiative transfer calculations, we limit the depth (along the sightline) of the gas ionized by the UVB to a value $L_{\max }$ defined by the condition that the column recombination equals the incident ionizing photon flux $\Phi_{\mathrm{i}}$ :

$$
\alpha_{B} \int_{0}^{L_{\max }}\left(n_{e} n_{\mathrm{H} \mathrm{II}}\right) d s \stackrel{!}{=} \Phi_{i}
$$

where the ionizing photon flux (in photons $\mathrm{cm}^{-2} \mathrm{~s}^{-1}$ ) is given by Equation (15). This condition implies that all the ionizing photons be absorbed within a depth $L_{\max }$, assuming the gas has been exposed to the (uniform) UV radiation field long enough to reach ionization equilibrium (which is well justified in the case of the Stream; see Bland-Hawthorn et al. 2013, their appendix). Note that this condition is equivalent to restricting the ionizing effect of the UVB to a column of gas $\sim 10^{17} \mathrm{~cm}^{-2}$. Indeed, using Equations (18) and (15), the condition (22) becomes (using $\gamma=1.8$ )

$$
\int_{0}^{L_{\max }} n_{\mathrm{HI}} d s=\frac{2}{3} N_{\mathrm{HI}}(\mathrm{LL}),
$$

where $N_{\mathrm{H} \text { I }}(\mathrm{LL}) \equiv \sigma_{0}^{-1} \approx 1.6 \times 10^{17} \mathrm{~cm}^{-2}$.

\section{REFERENCES}

Anderson, M. E., \& Bregman, J. N. 2010, ApJ, 714, 320

Asplund, M., Grevesse, N., Sauval, A. J., \& Scott, P. 2009, ARA\&A, 47, 481 Baker, D. J., \& Romick, G. J. 1976, ApOpt, 15, 1966 
Barger, K., Madsen, G. J., Fox, A., et al. 2015, in American Astronomical Society Meeting Abstracts, 225, \#253

Begelman, M. C., \& Fabian, A. C. 1990, MNRAS, 244, 26P

Besla, G., Kallivayalil, N., Hernquist, L., et al. 2007, ApJ, 668, 949

Besla, G., Kallivayalil, N., Hernquist, L., et al. 2012, MNRAS, 421, 2109

Binney, J., \& Tremaine, S. 2008, Galactic Dynamics (2nd ed.; Princeton, NJ: Princeton Univ. Press)

Bland-Hawthorn, J. 2009, in IAU Symp., The Galaxy Disk in Cosmological Context, ed. J. T. Van Loon \& J. M. Oliveira (Cambridge: Cambridge Univ. Press), 122

Bland-Hawthorn, J., Maloney, P. R., Sutherland, R. S., \& Madsen, G. J. 2013, ApJ, 778, 58

Bland-Hawthorn, J., Sutherland, R., Agertz, O., \& Moore, B. 2007, ApJL, 670, L109

Bland-Hawthorn, J., Veilleux, S., Cecil, G. N., et al. 1998, MNRAS, 299, 611

Blitz, L., \& Robishaw, T. 2000, ApJ, 541, 675

Bregman, J. N., Otte, B., Irwin, J. A., et al. 2009, ApJ, 699, 1765

Cavaliere, A., \& Fusco-Femiano, R. 1976, A\&A, 49, 137

Dickey, J. M., \& Lockman, F. J. 1990, ARA\&A, 28, 215

Dieter, N. H. 1971, A\&A, 12, 59

Einasto, J. 1965, TrAlm, 5, 87

Fox, A. J., Bordoloi, R., Savage, B. D., et al. 2015, ApJL, 799, L7

Fox, A. J., Richter, P., Wakker, B. P., et al. 2013, ApJ, 772, 110

Fox, A. J., Wakker, B. P., Barger, K. A., et al. 2014, ApJ, 787, 147

Fox, A. J., Wakker, B. P., Savage, B. D., et al. 2005, ApJ, 630, 332

Gao, L., Navarro, J. F., Cole, S., et al. 2008, MNRAS, 387, 536

Gatto, A., Fraternali, F., Read, J. I., et al. 2013, MNRAS, 433, 2749

Graczyk, D., Pietrzyński, G., Thompson, I. B., et al. 2014, ApJ, 780, 59

Guglielmo, M., Lewis, G. F., \& Bland-Hawthorn, J. 2014, MNRAS, 444, 1759

Guo, F., \& Mathews, W. G. 2012, ApJ, 756, 181

Haffner, L. M., Reynolds, R. J., Tufte, S. L., et al. 2003, ApJS, 149, 405

Heitsch, F., \& Putman, M. E. 2009, ApJ, 698, 1485

Henley, D. B., \& Shelton, R. L. 2013, ApJ, 773, 92

Henley, D. B., \& Shelton, R. L. 2014, ApJ, 784, 54

Hodges-Kluck, E. J., \& Bregman, J. N. 2013, ApJ, 762, 12

Ibata, R. A., Gilmore, G., \& Irwin, M. J. 1994, Natur, 370, 194

Kafle, P. R., Sharma, S., Lewis, G. F., \& Bland-Hawthorn, J. 2014, ApJ, 794, 59

Kallivayalil, N., van der Marel, R. P., Besla, G., Anderson, J., \& Alcock, C. 2013, ApJ, 764, 161

King, I. R. 1966, AJ, 71, 64

Konz, C., Lesch, H., Birk, G. T., \& Wiechen, H. 2001, ApJ, 548, 249

Kramida, A., Ralchenko, Y., Reader, J. \& NIST ASD Team 2014, NIST Atomic Spectra Database (ver. 5.2), NIST Atomic Spectra Database (ver. 5.2), [Online]. Available: http://physics.nist.gov/asd [2015, February 16]. National Institute of Standards and Technology, Gaithersburg, MD

Liska, R., \& Wendroff, B. 2003, SIAM Journal on Scientific Computing, 25,995

Lockman, F. J., Benjamin, R. A., Heroux, A. J., \& Langston, G. I. 2008, ApJL, 679, L21

Lu, L., Savage, B. D., \& Sembach, K. R. 1994, ApJL, 437, L119

Madsen, G. J. 2012, in EAS Publications Ser. 56, ed. M. A. de Avillez (Les Ulis: EDP Sciences), 281

Marinacci, F., Binney, J., Fraternali, F., et al. 2010, MNRAS, 404, 1464
Mathewson, D. S., Cleary, M. N., \& Murray, J. D. 1974, ApJ, 190, 291

McClure-Griffiths, N. M., Madsen, G. J., Gaensler, B. M., McConnell, D., \& Schnitzeler, D. H. F. M. 2010, ApJ, 725, 275

Miller, M. J., \& Bregman, J. N. 2013, ApJ, 770, 118

Miller, M. J., \& Bregman, J. N. 2015, ApJ, 800, 14

Navarro, J. F., Frenk, C. S., \& White, S. D. M. 1996, ApJ, 462, 563

Nichols, M., Mirabal, N., Agertz, O., Lockman, F. J., \& Bland-Hawthorn, J. 2014, MNRAS, 442, 2883

Nidever, D. L., Majewski, S. R., \& Burton, W. B. 2008, ApJ, 679, 432

Nidever, D. L., Majewski, S. R., Butler Burton, W., \& Nigra, L. 2010, ApJ, 723,1618

Oort, J. H. 1970, A\&A, 7, 381

Ostheimer, J. C., Majewski, S. R., \& Kunkel, W. E. 1997, in Bulletin of the American Astronomical Society 29, BAAS, 29, 1108

Pequignot, D., Petitjean, P., \& Boisson, C. 1991, A\&A, 251, 680

Planck Collaboration, Ade, P. A. R., Aghanim, N., et al. 2014, A\&A, 571, A16

Putman, M. E., Bland-Hawthorn, J., Veilleux, S., et al. 2003, ApJ, 597, 948

Putman, M. E., Gibson, B. K., Staveley-Smith, L., et al. 1998, Natur, 394, 752

Putman, M. E., Thom, C., Gibson, B. K., \& Staveley-Smith, L. 2004, ApJL, 603, L77

Rasmussen, J., Sommer-Larsen, J., Pedersen, K., et al. 2009, ApJ, 697, 79

Reynolds, R. J., Tufte, S. L., Haffner, L. M., Jaehnig, K., \& Percival, J. W. 1998, PASA, 15, 14

Richter, P., Fox, A. J., Wakker, B. P., et al. 2013, ApJ, 772, 111

Richter, P., Sembach, K. R., Wakker, B. P., \& Savage, B. D. 2001, ApJL, 562, L181

Salem, M., Besla, G., Bryan, G., et al. 2015, arXiv:1507.07935

Sembach, K. R., Wakker, B. P., Savage, B. D., et al. 2003, ApJS, 146, 165

Shull, J. M. 2014, ApJ, 784, 142

Shull, J. M., Moloney, J., Danforth, C. W., \& Tilton, E. M. 2015, arXiv: 1502.00637

Shull, J. M., Roberts, D., Giroux, M. L., Penton, S. V., \& Fardal, M. A. 1999, AJ, 118, 1450

Snowden, S. L., Freyberg, M. J., Kuntz, K. D., \& Sanders, W. T. 2000, ApJS, 128,171

Spitzer, L., Jr. 1956, ApJ, 124, 20

Stanimirović, S., Dickey, J. M., Krčo, M., \& Brooks, A. M. 2002, ApJ, 576,773

Sternberg, A., McKee, C. F., \& Wolfire, M. G. 2002, ApJS, 143, 419

Su, M., Slatyer, T. R., \& Finkbeiner, D. P. 2010, ApJ, 724, 1044

Sutherland, R. S. 2010, Ap\&SS, 327, 173

Sutherland, R. S., \& Bicknell, G. V. 2007, ApJS, 173, 37

Suto, Y., Sasaki, S., \& Makino, N. 1998, ApJ, 509, 544

Tufte, S. L. 1997, PhD thesis, The Univ. Wisconsin

Wakker, B. P. 2001, ApJS, 136, 463

Walker, A. R. 2012, Ap\&SS, 341, 43

Wannier, P., \& Wrixon, G. T. 1972, ApJL, 173, L119

Wegg, C., Gerhard, O., \& Portail, M. 2015, MNRAS, 450, 4050

Weiner, B. J., Vogel, S. N., \& Williams, T. B. 2002, in ASP Conf. Ser. 254, Extragalactic Gas at Low Redshift, ed. J. S. Mulchaey \& J. T. Stocke (San Francisco, CA: ASP), 256

Weiner, B. J., \& Williams, T. B. 1996, AJ, 111, 1156

Werk, J. K., Prochaska, J. X., Tumlinson, J., et al. 2014, ApJ, 792, 8

Weymann, R. J., Vogel, S. N., Veilleux, S., \& Epps, H. W. 2001, ApJ, 561,559 